\title{
On the Exact Inter-departure, Inter-start, and Cycle Time Distribution of Closed Queueing Networks Subject to Blocking
}

\author{
September 30, 2014 \\ Svenja Lagershausen \\ Institut für Produktionswirtschaft, Leibniz Universität Hannover, Hannover, Germany, \\ Email: svenja.lagershausen@prod.uni-hannover.de Phone: +49511762 2982 Fax: +49 5117624863 \\ Barış Tan \\ College of Administrative Sciences and Economics, Koç University, Rumeli Feneri Yolu, Istanbul, Turkey, 34450 \\ Email: btan@ku.edu.tr Phone: +90 2123381459 Fax: +90 2123381653
}

This paper presents a method to determine the exact inter-departure, inter-start and cycle time distribution of closed queueing networks that can be modeled as Continuous-Time Markov Chains with finite state space. The method is based on extending the state space to determine the transitions that lead to a departure or to an arrival of a part on a station. Once these transitions are identified and represented in an indicator matrix, a first passage time analysis is utilized to determine the exact distributions of the inter-departure, interstart, and cycle time. In order to demonstrate the methodology, we consider closed-loop production lines with phase-type service time distributions and finite buffers. We discuss the methodology to automatically generate the state space and to obtain the transition rate matrices for the considered distributions. We use the proposed method to analyze the effects of the system parameters on the inter-departure, inter-start time, and cycle time distributions numerically for various cases. The proposed methodology allows the exact analysis of the inter-departure, inter-start, and cycle time distributions of a wide range of production systems with phase-type servers that can be modeled as Continuous-Time Markov Chains in a unified way.

Key words: Inter-departure Time, Cycle Time, Inter-start Time, Phase-type Distribution, Closed

Queueing Networks, First Passage Time Analysis

\section{Introduction}

In this paper, we present a method to determine the exact inter-departure, inter-start, and cycle time distributions of closed queueing networks subject to blocking that can be modeled as Continuous-Time Markov Chains (CTMC) with a finite state space. Queueing networks are commonly used for modeling production and telecommunication systems. The inter-departure time distribution gives valuable information about the variability of the output from a production system. Similarly, the flow variability in a queueing network can be analyzed by studying the interstart time distributions. The cycle time distribution reveals information about the variability of 
the time a customer spends in the queueing network. Although there are numerous studies on the performance analysis of production systems to determine their average performance measures, the number of studies that consider the variability of the output, the variability of the cycle time, and the variability of flow is limited. Although the importance of studying these variabilities is noted in the literature, analytical tractability is limited mainly due to the fact that the processes (e.g. departure process) are not renewal in general queueing networks, see Wu and McGinnis (2013) for a discussion on non-renewal departure processes. The special structure of the class of the queueing systems analyzed in this study, namely Closed Queueing Networks (CQN) with phase-type servers, allows us to analyze the inter-start, cycle time, and output variability analytically.

Furthermore, many approximation methods that are based on decomposition use simplified inter-departure time distributions of the flows in two-station subsystems that capture the inflow and outflow dynamics in the station parameters. The method presented in this study allows us to determine the exact distribution, and therefore, can be used to evaluate the accuracy of approximations used for cycle, inter-start, and inter-departure time distributions.

The method presented in this paper is based on expressing the inter-departure, inter-start, and cycle time as the first passage time in a process that is generated from the original CTMC. In the new process, the entry and exit states corresponding to the flow of a part between two specific points in time are identified depending on the inter-event time of interest. After determining the state of the system when a part completes its operation on a given station, the first passage time to one of the states corresponding to the next part completion on the same station gives the interdeparture time distribution. Similarly, identifying the state of the system in which a part starts its operation on a given station, the first passage time to one of the states corresponding to the next processing start on the same station gives the inter-start time distribution. The cycle time is also represented as a first passage time from the initial states where an arriving part starts its operation to the final states where the same part finishes its operation at the last station. This is done by extending the state space to track the position of each part on each station until the work-in-process an arriving part sees in the system is depleted and the same part is processed at the final station.

This general approach allows us to develop a two-stage method. In the first stage, we generate the state space model of a given queueing network automatically. In the second stage, we use this state space model to obtain the extended state space models for the inter-departure, inter-start, and cycle-time distribution, and determine the exact distributions by using the first-passage time analysis. The first stage is described in Lagershausen (2012). This paper focuses on the description of the second stage. 
Since the method is a state-space-based method, it is affected by rapid increase of states with the number of stations and the buffer capacities as all the other state-space-based methods. Still, its ability to determine the exact inter-departure, inter-start, and cycle time distributions of closed queueing models that can be modeled as CTMCs is its advantage compared to other methods found in the literature.

The organization of the remaining part of this paper is as follows. In Section 2, the pertinent literature is reviewed. The methodology is presented in Section 3. In Section 4, an example of a closed-loop production system with three exponential servers and a given number of pallets is used to describe the methodology. Models of production systems with Coxian-2, general Coxian, and Erlang servers are also discussed in this section. Numerical results are presented in Section 5 . Finally, conclusions are given in Section 6 .

\section{Literature Review}

Both the inter-departure time and the cycle time are related to the variability of flow and the variability of output in a production system. In the literature, there are various studies that focus on either the inter-departure time or the cycle time distribution. Since our method gives the exact distributions of the inter-departure, inter-start, and cycle time, we review the most relevant studies that focus on each of these inter-event distributions.

\subsection{Inter-departure Time Distribution}

Duenyas and Hopp (1990) developed an approximation for the output-variance in closed queueing systems with exponential processing times and unlimited buffer capacity. Duenyas, Hopp, and Spearman (1993) proposed an approximation for CQN with deterministic processing times and geometrically distributed outages. These two approaches are the only methods for higher-order moments of the inter-departure-time in closed queueing systems. However, there are further methods for open queueing systems.

Miltenburg (1987) determined the asymptotic variance rate. Hendricks (1992) derived the marginal probability function for the inter-departure time of exponential, finite-buffered open queueing systems using Markov chains. Hendricks and McClain (1993) investigated the influence of the input parameters on the variance of the inter-departure time for general, finite-buffered open queueing systems by simulation. Gershwin (1993) presented an exact closed-form expression for the variance of the output of an unreliable station in a given time interval.

Kim and Alden (1997) studied single-server stations with deterministic processing times and exponentially distributed failure and repair times. They provided an analytical approximation for the density function and the variance of the time to produce a fixed lot size. Li and Meerkov 
Table 1 Methods for higher-order moments of the inter-departure-time or the output

\begin{tabular}{|c|c|c|c|c|c|c|c|c|c|}
\hline Authors & $\mathrm{C}$ & $\mathrm{M}$ & $\mathrm{S}$ & DP & $\mathrm{DF}$ & DR & BS & $\mathrm{BM}$ & $\mathrm{R}$ \\
\hline Buzacott, Liu, and Shanthikumar (1995) & OL & 2 & $\mathrm{~S}$ & G & - & - & $\mathrm{L}$ & BAS & app \\
\hline Duenyas and Hopp (1990) & CL & M & $\mathrm{S}$ & $\mathrm{E}$ & - & - & $\mathrm{U}$ & - & app \\
\hline Duenyas, Hopp, and Spearman (1993) & CL & $\mathrm{M}$ & $\mathrm{S}$ & $\mathrm{D}$ & $\mathrm{E}$ & $\mathrm{E}$ & $\mathrm{U}$ & - & app \\
\hline Gershwin (1993) & OL & 2 & $\mathrm{~S}$ & $\mathrm{D}$ & Geo & Geo & 0 & - & ex \\
\hline Gelenbe (1975) & $\mathrm{OL}$ & 2 & $\mathrm{~S}$ & G & - & - & $\mathrm{U}$ & - & app \\
\hline Hendricks (1992) & OL & $\mathrm{M}$ & $\mathrm{S}$ & $\mathrm{E}$ & - & - & $\mathrm{L}$ & BAS & ex \\
\hline Hendricks and McClain (1993) & OL & M & $\mathrm{S}$ & G & - & - & $\mathrm{L}$ & BAS & app \\
\hline Kim and Alden (1997) & OL & 1 & $\mathrm{~S}$ & $\mathrm{D}$ & $\mathrm{E}$ & $\mathrm{E}$ & - & - & app \\
\hline Li and Meerkov (2000) & OL & M & $\mathrm{S}$ & $\mathrm{D}$ & Geo & $\mathrm{D}$ & $\mathrm{L}$ & & app \\
\hline Manitz (2005) & $\mathrm{OA}$ & M & $\mathrm{S}$ & G & - & - & $\mathrm{L}$ & BAS & app \\
\hline Manitz and Tempelmeier (2012) & OD & $\mathrm{M}$ & $\mathrm{S}$ & G & - & - & $\mathrm{L}$ & BAS & app \\
\hline Miltenburg,(1987) & OL & 3 & $\mathrm{~S}$ & $\mathrm{D}, \mathrm{Geo}$ & Geo & Geo & $\mathrm{L}$ & BAS & app \\
\hline $\operatorname{Tan}(1999 \mathrm{a}$ & OL & M & $\mathrm{S}$ & $\mathrm{D}$ & Geo & Geo & 0 & - & ex \\
\hline $\operatorname{Tan}(1999 \mathrm{~b})$ & OL & 2 & $\mathrm{~S}$ & $\mathrm{D}$ & Geo & Geo & $\mathrm{L}$ & & ex \\
\hline $\operatorname{Tan}(2000)$ & OL & 2,3 & $\mathrm{~S}$ & D & Geo & Geo & $\mathrm{L}$ & & ex \\
\hline
\end{tabular}

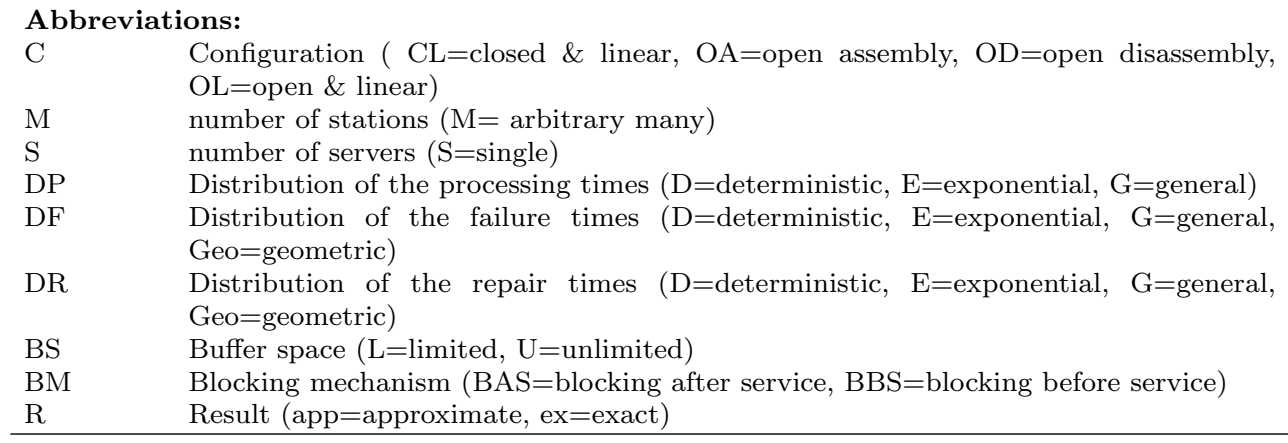

(2000) assumed stations with Bernoulli distributions. They developed bounds for the variability of the output.

$\operatorname{Tan}(1998)$ proposed an analytical formula for the variance of the inter-departure time for open queueing systems with no interstage buffers, time-dependent failures, and continuous material flow. Tan (1999a) and Tan (1999b) presented an exact closed-form expression for the variance of the output in a given time interval assuming a general two-station production line with finite buffer capacities. For this, a Markov reward model was set up and equations were recursively solved. Tan (2000) proposed a numerical exact approach for the output-variance of open queueing systems with deterministic processing times and geometrically distributed failure and repair times and two or three stations. $\operatorname{Tan}(2013)$ gives a review of recent studies on the variance of the output for open queueing systems and summarizes the analytical results.

The literature contains, moreover, several approximations for the coefficient of variation of the inter-departure time in queueing models. Gelenbe (1975) proposed an estimation under the assumption of general processing times and infinite buffer spaces using the diffusion approximation. Buzacott, Liu, and Shanthikumar (1995), Manitz (2005), and Manitz and Tempelmeier (2012) considered queueing models with general processing times and finite buffer spaces. Buzacott, Liu, and Shanthikumar (1995) considered two-station linear systems, Manitz (2005) assumed twostation assembly systems, and Manitz and Tempelmeier (2012) studied two-station disassembly systems. Table 1 gives an overview of the literature. 


\subsection{Inter-Start Time Distribution}

Lagershausen (2012) proposed a formulation of the inter-start time distribution for closed queueing networks with phase-type distributed processing times and finite buffers using a smaller state space, however restricted to the inter-start time. We are not aware of any other studies that focus on the inter-start time distribution.

\subsection{Cycle Time Distribution}

Chow (1980) determined the cycle time distribution for a two-stage CQN with exponential processing times and infinite buffers. Following Chow, several more papers on the cycle time distribution in CQN with single servers were produced (Schassberger and Daduna (1983), Boxma and Donk (1982), Boxma, Kelly, and Konheim (1984), Daduna (1982), Kelly and Pollett (1983)). Boxma (1983) derived an expression for the Laplace-Stieltjes transform of the distribution of the cycle time distribution for a two-station CQN. Using one exponential and one general server, he showed that the distribution of the cycle time depends on the order in which two stations are visited. As stated there, the cycle times are dependent. This is also the reason for the aforementioned result. In a more recent paper, Ayhan, Palmoski, and Schlegel (2004) provide bounds for the $n$ th departure time assuming multiple stages, sub-exponential service times, and infinite buffers. Shi and Gershwin (2011) propose an analytical expression for the waiting time distribution in a two-station system in discrete time subject to failures.

\section{Methodology}

We consider a closed queueing network subject to blocking that can be modeled as a ContinuousTime Markov Chain. There are $K$ stations. Each station has a finite input buffer. The processing time on each station has a phase-type distribution.

Based on the data collected on the service times from manufacturing systems, Inman (1999) shows that service times have a variability lower than one. In order to model distributions with a coefficient of variation unequal to one and additionally to carry out an exact analysis, we use phasetype distributions. These distributions can be used to approximate a wide range of distributions (Neuts 1995).

The state of the system at time $t$ is $X(t) \in S . X(t)$ is a $K$-tuple where each element shows the number of parts on a given station including its input buffer, its phase, and whether it is blocked or not. Since the processing times have phase-type distributions, the process $\{X(t), t \geq 0\}$ is a Continuous-Time Markov Chain. The corresponding infinitesimal generator or the rate matrix of the process $\{X(t), t \geq 0\}$ is denoted with $Q=\left\{q_{i, j}\right\}$. For $i \neq j, q_{i, j}$ is the transition rate from state $i$ to state $j$. Since a given number of parts circulate in the system, all states constitute a single 
communicating class and, therefore, steady-state exists.

We consider the distributions of three different times between events: the departure of a part from a station (inter-departure time), the start of the processing at a station (inter-start time), and the departure of a part from the same station (cycle time). We determine the distributions of the time between events from the first passage time distribution. In the next section, we present a general methodology that is used for the inter-departure, inter-start, and the cycle time distribution. The methodology is demonstrated in Section 4 by using a specific production system with the state transition diagrams for the original and the extended processes. The reader may find it easier to follow the methodology in Sections 3.1 to 3.4 in parallel with the example in Section 4 .

\subsection{First Passage Time Analysis for the Distributions of the Inter-event Times}

In our methodology we define a new process based on $\{X(t), t \geq 0\}$ to identify transitions that lead to a departure from a station, to a processing start at a station or to the completion of the process at the last station. In order to identify these transitions, the original state space $S$ is duplicated. That is the same state $i$ in $S$ appears twice for inter-departure and inter-start time distributions; and it appears exactly the same number of times as the number of parts in front of an arriving part for the cycle-time distributions. We then analyze the new process defined on an extended state space that includes the states after a departure, a processing start, or completion of a part.

The new process is denoted by $\left\{X_{e}^{\prime}(t), t \geq 0\right\}$ where $e \in\{d, s, c\}$; and $d$ is used for inter-departure time $T_{d}, s$ is used for inter-start time $T_{s}$, and $c$ is used for cycle time $T_{c}$.

The process $\left\{X_{e}^{\prime}(t), t \geq 0\right\}$ includes entry states that are transient and exit states that are absorbing states. The process starts at one of the entry states where the inter-event time starts and ends at one of the exit states where the current event of interest that is departure, arrival, or completion of a cycle time ends. The next event will start from this state that will be the entry state for the analysis of the next cycle. In this representation, the inter-event time is the first-passage time from an initial state to one of the exit states.

The rate matrix of the process $\left\{X_{e}^{\prime}(t), t \geq 0\right\}$ is denoted by $Q_{e}^{\prime}, e \in\{d, s, c\}$. When the states of the process $\left\{X_{e}^{\prime}(t), t \geq 0\right\}$ are ordered as the transient states are followed by the absorbing states depending on the type of the event, the rate matrix $Q_{e}^{\prime}$ can be put in the form

$$
Q_{e}^{\prime}=\left[\begin{array}{c|c}
Q_{e} & R_{e} \\
\hline 0 & 0
\end{array}\right]
$$

where $Q_{e}$ is the infinitesimal generator submatrix for the transitions from the transient states to other transient states, $R_{e}$ is the submatrix with elements that are the transition rates from the transient states to the absorbing states, and 0s are zero matrices of appropriate sizes.

In our approach, the transitions that lead to the event of interest (e.g. a departure, processing 
start, or cycle completion) are identified and represented in an indicator matrix $G_{e}$. The matrices $Q_{e}$ and $R_{e}$ in Equation (1) are determined directly from the state transition matrix of the original process $Q$ and the indicator matrix $G_{e}$. In the next sections, we discuss how the indicator matrices are determined for the inter-departure, inter-start, and cycle time distributions.

By using the steady-state probabilities of entering the entry states as the initial probabilities, the inter-event time distribution can be determined from the steady-state first passage time distribution.

The initial probability vector with its $i$ th element being the probability that the process starts at state $i$ at time $\tau$ is denoted with $\pi_{e}^{\text {entry }}(\tau), e \in\{d, s, c\}$. Similarly, the probability vector with its $i$ th element being the probability that the process is at state $i$ at time $t+\tau$ is denoted with $\pi_{e}^{\text {exit }}(t+\tau), e \in\{d, s, c\}$.

The probability vectors $\pi_{e}^{\text {entry }}(\tau)$ and $\pi_{e}^{\text {exit }}(t+\tau)$ satisfy the following differential equation:

$$
\frac{d}{d t} \pi_{e}^{\text {exit }}(t+\tau)=\pi_{e}^{\text {entry }}(\tau) e^{Q_{e} t} R_{e}, \quad e \in\{d, s, c\} .
$$

Let $\pi_{e}^{\text {entry }}=\lim _{\tau \rightarrow \infty} \pi_{e}^{\text {entry }}(\tau)$ and $\pi_{e}^{\text {exit }}=\lim _{\tau \rightarrow \infty} \pi_{e}^{\text {exit }}(\tau)$ be the steady state probabilities. In the steadystate, the steady-state probabilities $\pi_{e}^{\text {entry }}$ and $\pi_{e}^{\text {exit }}$ satisfy

$$
\pi_{e}^{\mathrm{exit}}=-\pi_{e}^{\mathrm{entry}} Q_{e}^{-1} R_{e}, \quad e \in\{d, s, c\} .
$$

The transition from one of the entry states to one of the exit states represents a new inter-event cycle. In other words, we consider events that take place one after the other in a recurring way from an entry state to an exit state, and then from this state that becomes an entry state to another exit state and so on. However, we break this event cycle into inter-event cycles. This implies that these exit states in the new event cycle are in fact the same as the entry states in the current cycle. For example, Figure 2 shows the state transition diagram of the process that captures the production of parts on Station 1 for two succeeding inter-departure time cycles for the specific example of a 3-station production line that will be discussed in Section 4

Consequently, the steady-state probabilities of entering the entry states are equal to the steadystate probabilities of absorption in one of the exit states. Then setting $\pi_{e}^{\text {entry }}=\pi_{e}^{\text {exit }}$ in Equation (3) yields

$$
\pi_{e}^{\text {entry }}\left(I+Q_{e}^{-1} R_{e}\right)=0, \quad e \in\{d, s, c\} .
$$

Since the process enters in one of the states,

$$
\pi_{e}^{\text {entry }} u=1, \quad e \in\{d, s, c\}
$$

where $u$ is a column vector of ones. Then, $\pi_{e}^{\text {entry }}$ is obtained by solving Equations (4) and (5). 
The steady-state inter-event time probability distribution $F_{T_{e}}(t)$ is given as

$$
F_{T_{e}}(t)=1-\pi_{e}^{\text {entry }} e^{Q_{e} t} u, \quad e \in\{d, s, c\}
$$

and the steady-state probability density function of the inter-event time is given as

$$
f_{T_{e}}(t)=\frac{d}{d t} F_{T_{e}}(t)=-\pi_{e}^{\text {entry }} Q_{e} e^{Q_{e} t} u, \quad e \in\{d, s, c\} .
$$

The mean and the variance of the inter-event time, $E\left[T_{e}\right]$ and $\operatorname{Var}\left[T_{e}\right], e \in\{d, s, c\}$ are also obtained directly from the matrices $Q_{e}$ and $R_{e}$ :

$$
\begin{gathered}
E\left[T_{e}\right]=-\pi_{e}^{\text {entry }} Q_{e}^{-1} u, \quad e \in\{d, s, c\} \\
\operatorname{Var}\left[T_{e}\right]=2 \pi_{e}^{\text {entry }} Q_{e}^{-2} u-\left(\pi_{e}^{\text {entry }} Q_{e}^{-1} u\right)^{2}, \quad e \in\{d, s, c\} .
\end{gathered}
$$

In the next section, we will define the indicator matrices $G_{d}, G_{s}$, and $G_{c}$ for inter-departure, inter-start, and cycle time distributions, respectively. Once the indicator matrices are determined, the methodology presented in this section yields the exact distribution of the inter-departure, inter-start and cycle time.

\subsection{Inter-departure Time Distribution}

The first passage time of the inter-departure time distribution is the time from the instant a part departs from a station to the instant the next part departs from the same station. Note that the inter-departure time distribution depends on the station where the output is observed. So, in order to compute the inter-departure time distribution, we use Station $k$ as the designated station without loss of generality.

In order to define the inter departure time formally, we define a counting process for station $k,\left\{N_{d_{k}}(t), t \geq 0\right\}, N_{d_{k}}(t)=0,1,2, \ldots$, associated with $\{X(t), t \geq 0\} . N_{d_{k}}(t)$ is the number of parts produced by Station $k$ in the time period $[0, t)$. The time instance $N_{d_{k}}(t)$ increases from $n$ to $n+1$ is denoted with $t_{d_{k, n+1}}$. Considering the counting process, the inter-departure time of Station $k$ is the first-passage time from the state at time $t_{d_{k, n}}$ to the state at time $t_{d_{k, n+1}}$. The steady-state distribution of the inter-departure time is the distribution of $\lim _{n \rightarrow \infty}\left\{t_{d_{k, n+1}}-t_{d_{k, n}}\right\}$.

In order to use the first passage time analysis, we duplicate all the states in $S$ in the state space of the new process $\left\{X_{d}^{\prime}(t), t \geq 0\right\}$. In order to differentiate the duplicated states, we refer to them as states in $S^{*}$. As a result, the state space of $\left\{X_{d}^{\prime}(t), t \geq 0\right\}$ is $S \cup S^{*}$.

In the process $\left\{X_{d}^{\prime}(t), t \geq 0\right\}$, the inter-departure time starts in one of the states in $S$ where the process enters upon the completion of a part on station $k$; and it is absorbed in one of the states in $S^{*}$ where the process enters after the completion of a part on station $k$. As a result, no transition takes place from a state in $S^{*}$ to a state in $S$. 
In order to identify a departure event, let $G_{d}=\left\{g_{i, j}\right\}$ be an indicator matrix where $g_{i, j}$ is 1 if a transition from state $i$ to state $j$ increases $N_{d_{k}}(t)$ by one and it is 0 otherwise. The elements of $G_{d}=\left\{g_{i, j}\right\}$ are determined by checking certain conditions based on the states $i$ and $j$ of the original state space. Namely, for each tuple $(i, j)$ with a positive $q_{i, j}$,

- if a part completes its operation on station $k$ in state $i$ and it is not blocked in state $j$, or

- if station $k$ is blocked in state $i$ and it is not blocked in state $j$

then $g_{i, j}$ is 1 . Therefore, the matrix $G_{d}$ is completely specified by the rate matrix $Q$ and the state space $S$.

Once $G_{d}$ is available, the matrices are determined by using $G_{d}$ and the original generator matrix of the queueing network $Q$ as given below:

$$
R_{d}=Q \circ G_{d}
$$

where $\left(Q \circ G_{d}\right)_{i, j}=q_{i, j} g_{d_{i, j}}$ is the element-wise product (Schur product) of matrices $Q$ and $G_{d}$ and

$$
Q_{d}=Q-R_{d}
$$

The probability vector for entering the states, $\pi_{d}^{\text {entry }}$, is obtained by solving Equations (4) and (5) after plugging $Q_{d}$ and $R_{d}$ into these equations. With these expressions, the distribution function and probability density function of $T_{d}$, its expected value and variance are obtained from Equations (6) to (9).

\subsection{Inter-start Time Distribution}

The methodology described above for the inter-departure time distribution can be extended directly to the distribution of the inter-start time distribution by determining the appropriate indicator matrix $G_{s}$ that shows which transitions in the process $\{X(t), t \geq 0\}$ correspond to the processing start of a new part on Station $k$.

We first define the inter-start time formally. Associated with $\{X(t), t \geq 0\}$, we also define another counting process for Station $k,\left\{N_{s_{k}}(t), t \geq 0\right\}, N_{s_{k}}(t)=0,1,2, \ldots$ where $N_{s_{k}}(t)$ is the number of parts that started its process on Station $k$ in the time period $[0, t)$. The time instance $N_{s_{k}}(t)$ increases from $n$ to $n+1$ on Station $k$ is denoted with $t_{s_{k, n+1}}$. The steady-state distribution of the inter-start time is the distribution of $\lim _{n \rightarrow \infty}\left\{t_{s_{k, n+1}}-t_{s_{k, n}}\right\}$.

Similar to the previous case, our methodology for the inter-start time distribution is based on extending the original state space by identifying the transitions that lead to the start of processing at Station $k$. We define the indicator matrix for the inter-start time $G_{s}=\left\{g_{s_{i, j}}\right\}$ as $g_{s_{i, j}}$ is 1 if a transition from state $i$ to state $j$ increases $N_{s_{k}}(t)$ by one and it is 0 otherwise. 
The elements of $G_{s}=\left\{g_{s_{i, j}}\right\}$ are set to 1 or 0 according to whether the states $i$ and $j$ satisfy certain conditions. For each tuple $(i, j)$ with a positive $q_{i, j}$,

- if the number of parts at Station $k$ in state $i$ is greater than one and a part departs from Station $k$ with a transition to state $j$, or

- if the preceding station is blocked in state $i$ and the blocking is removed with a transition to state $j$, or

- if Station $k$ is starved in state $i$ and a part departs from the preceding station in state $j$

then $g_{s_{i, j}}$ is 1 . Therefore, the matrix $G_{s}$ is completely specified by the rate matrix $Q$ and the state space $S$.

The matrices $Q_{s}$ and $R_{s}$ that yield the inter-start time distribution following Equation (6) are determined by using $G_{s}$ and the original generator matrix of the queueing network $Q$ :

$$
R_{s}=Q \circ G_{s}
$$

where $\left(Q \circ G_{s}\right)_{i, j}=q_{i, j} g_{s_{i, j}}$ is the element-wise product of matrices $Q$ and $R$ and

$$
Q_{s}=Q-R_{s}
$$

As in the case of the inter-departure time, by plugging $Q_{s}$ and $R_{s}$ into Equations (4) and (5) and by solving those, the probability vector for entering the states, $\pi_{s}^{\text {entry }}$, is obtained. With these expressions, the distribution and probability density function of $T_{s}$, its expected value and variance are found as shown in Equations (6) to (9).

\subsection{Cycle Time Distribution}

The cycle time is the time span from the instant a part arrives at a particular station to the instant the same part departs from the preceding station. In the following, without loss of generality, we will consider the cycle time from the instant when a part enters Station 1 to the instant this part departs from the last station, Station $K$. Since there are $M$ parts circulating in the system, $M$ parts need to be processed before this part completes its cycle time. Since the time instance of an arrival at the first station is equivalent to the departure at the last station, the steady-state distribution of the cycle time is the distribution of $\lim _{n \rightarrow \infty}\left\{t_{d_{K, n+M}}-t_{d_{K, n}}\right\}$.

In order to use the first-passage-time analysis, we define a new process $\left\{X_{c}^{\prime}(t), t \geq 0\right\}$ to track the flow of $M$ parts in the work-in-process from the instant a new part arrives to the instant this part completes its operation at the last station. $\left\{X_{c}^{\prime}(t), t \geq 0\right\}$ is defined in a state space that duplicates all the states in $S, M$ times. We refer to the set of states when there are exactly $m$ parts in the network as $S_{m}, m=M, M-1, \cdots, 1$. 
The process starts in one of the states in $S_{M}$ where a new part starts its operation on Station 1 ; and it is absorbed in one of the states where the same part departs after receiving service on station $K$ in $S_{1}$. In between, the number of parts in the work-in-process in front of the considered part decreases as the part moves from Station 1 to $K$. As a result, no transition from a state in $S_{m-1}$ to a state in $S_{m}$ takes place, $\forall m=M, M-1, \cdots, 2$.

The rate matrix of the process $\left\{X_{c}^{\prime}(t), t \geq 0\right\}$ is a $M n(Q) \times M n(Q)$ matrix $Q_{c}^{\prime}$. When the states of the process $\left\{X_{c}^{\prime}(t), t \geq 0\right\}$ are ordered as $\left\{S_{M}, S_{M-1}, \cdots, S_{1}\right\}$, the rate matrix $Q_{c}^{\prime}$ can be put in the form

$$
Q_{c}^{\prime}=\left[\begin{array}{c|c}
Q_{c} & R_{c} \\
\hline 0 & 0
\end{array}\right]
$$

where zeroes are row vectors of the appropriate size: The matrices $Q_{c}$ and $R_{c}$ are of the following structure:

$$
\begin{gathered}
Q_{c}=\left[\begin{array}{cccccc}
\tilde{Q}_{c} & \tilde{R}_{c} & 0 & \cdots & 0 & 0 \\
0 & \tilde{Q}_{c} & \tilde{R}_{c} & \cdots & 0 & 0 \\
\cdot & \cdot & . & \cdots & . & . \\
0 & 0 & 0 & \cdots & \tilde{Q}_{c} & \tilde{R}_{c} \\
0 & 0 & 0 & \cdots & 0 & \tilde{Q}_{c}
\end{array}\right] \\
R_{c}=\left[\begin{array}{cccccc}
0 & 0 & 0 & \cdots & 0 & 0 \\
0 & 0 & 0 & \cdots & 0 & 0 \\
. & . & . & \cdots & . & . \\
0 & 0 & 0 & \cdots & 0 & 0 \\
\tilde{R}_{c} & 0 & 0 & \cdots & 0 & 0
\end{array}\right]
\end{gathered}
$$

In the above matrices, $\tilde{Q}_{c}$ is a $n(Q) \times n(Q)$ infinitesimal generator submatrix for the transitions from the states in $S_{m}$ to the states in $S_{m}$ and $\tilde{R}_{c}$ is a $n(Q) \times n(Q)$ submatrix with elements that are the transition rates from the states in $S_{m}$ to the states $S_{m-1}, \forall m=M, M-1, \cdots, 1$, and zeroes appear in the matrices $Q_{c}$ and $R_{c}$ area of the same size as $\tilde{Q}_{c}$ and $\tilde{R}_{c}$.

Since the number of parts in the network decreases with the departures from Station $K$, we use an indicator matrix denoted with $G_{c}=\left\{g_{c_{i, j}}\right\}$ where $g_{c_{i, j}}$ is 1 if a transition from state $i$ to state $j$ increases the departures on Station $K$ by one, or equivalently decreases the number of parts in the network by one. Similar to the previous cases, $G_{c}$ is constructed by checking whether the states $i$ and $j$ of the state space satisfy certain conditions. For each tuple $(i, j)$ with a positive $q_{i, j}$,

- if a part completes its operation on station $K$ in state $i$ and it is not blocked in state $j$, or

- if station $K$ is blocked in state $i$ and it is not blocked in state $j$

then $g_{c_{i, j}}$ is 1 . Therefore, the matrix $G_{c}$ is completely specified by the rate matrix $Q$ and the state space $S$.

The matrices $\tilde{R}_{c}$ and $\tilde{Q}_{c}$, that are used to construct $Q_{c}^{\prime}$ and $R_{c}^{\prime}$ according to Equations (14) and (15), are determined by using $Q$ and $G_{c}$ as

$$
\tilde{R}_{c}=Q \circ G_{c}
$$


where $\left(Q \circ G_{c}\right)_{i, j}=q_{i, j} g_{c_{i, j}}$ is the element-wise product (Schur product) of matrices $Q$ and $R$ and

$$
\tilde{Q}_{c}=Q-\tilde{R}_{c}
$$

As for the inter-departure time distribution and the inter-start time distribution, the distribution and probability density function of $T_{c}$, its expected value and variance are obtained by using $Q_{c}$ and $R_{c}$ in Equations (4) to (9).

The state-space model of a given queueing network yields the matrices $Q_{d}$ and $R_{d}, Q_{s}$ and $R_{s}, Q_{c}$ and $R_{c}$, automatically for a given system. Given those matrices, the inter-departure, the inter-start time, and the cycle time distribution is obtained in a unified way as presented in this section. In the next section, we show an example of a three-station production line to explain our methodology.

\section{An Example}

In order to demonstrate the methodology, we analyze a closed-loop three-station system with servers that have exponentially distributed service times and $M=3$ parts circulating in the system. There is a buffer of size $b_{i}=1$ in front of each station. The processing rate of Station $i$ is $\mu_{i}$.

\section{1. $\quad$ State Space Model}

The state of the system is a 3 -tuple $X(t)=\left(X_{1}, X_{2}, X_{3}\right)$ where $X_{k}$ is the state of Station $k$, $k=1,2,3 . X_{k}$ gives the number of parts at Station $k$ including its input buffer and also indicates whether it is blocked with the number of parts at the station. For example $X_{1}=2$ indicates that there is one part being processed at station 1 and another part in its input buffer; $B_{1}(1)$ indicates that station 1 is blocked with the part that has completed its operation on station 1 and its input buffer is empty.

When there are three pallets circulating in the system, with a buffer of size one at each station, there are the following ten states in the state space:

$$
S=\left\{(2,1,0),(2,0,1),\left(2,0, B_{3}(1)\right),(1,2,0),(1,1,1),(1,0,2),(0,2,1),(0,1,2),\left(B_{1}(1), 2,0\right),\left(0, B_{2}(1), 2\right)\right\}
$$

Figure 1 shows the state space model for this case and Table 2 gives the transition rate matrix $Q$.

\subsection{Inter-departure Time Distribution}

In order to determine the inter-departure time distribution, we define the new process by duplicating the state space $S$. Figure 2 shows the state transition diagram of the process that captures the production of parts on Station 1 for two succeeding inter-departure time cycles. The two 
Figure 1 State Transition Diagram of $\{X(t), t \geq 0\}$ associated with the model of a closed three-station production line with exponential servers and 3 pallets

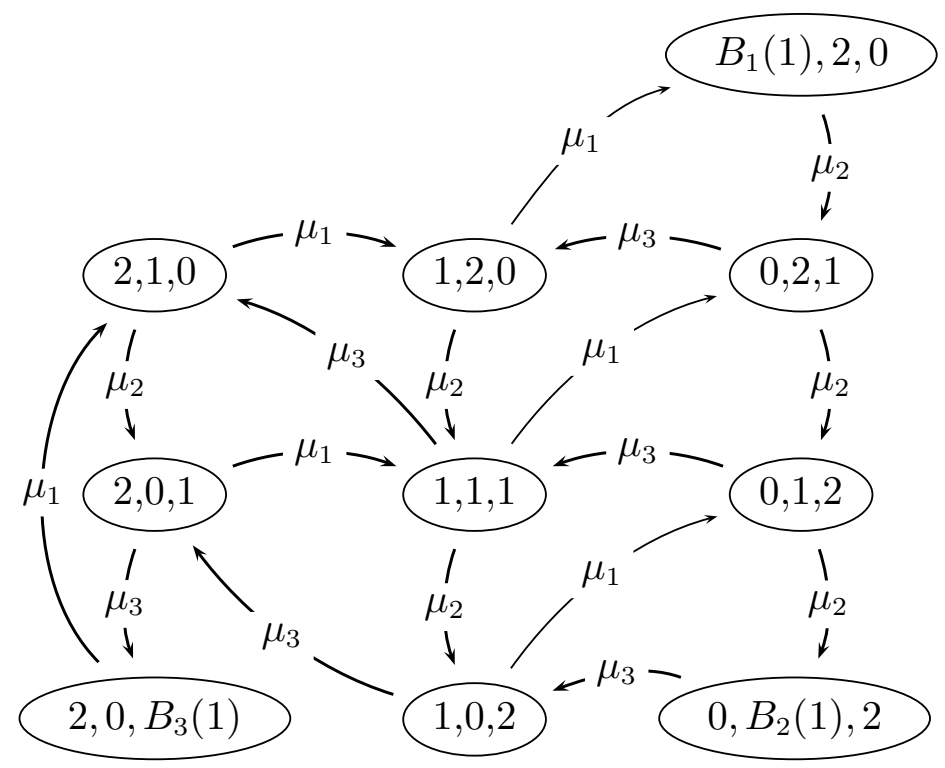

Table 2 Transition rate matrix $Q$ for the Markov chain model of a closed three-station production line with

\begin{tabular}{l|llllllllll|}
\multicolumn{10}{c}{ exponential servers and 3 pallets } \\
& $(2,1,0)$ & $(2,0,1)$ & $\left(2,0, B_{3}(1)\right)$ & $(1,2,0)$ & $(1,1,1)$ & $(1,0,2)$ & $(0,2,1)$ & $(0,1,2)$ & $\left(B_{1}(1), 2,0\right)\left(0, B_{2}(1), 2\right)$ \\
\hline$(2,1,0)$ & $-\mu_{1}-\mu_{2}$ & $\mu_{2}$ & 0 & $\mu_{1}$ & 0 & 0 & 0 & 0 & 0 & 0 \\
$(2,0,1)$ & 0 & $-\mu_{1}-\mu_{3} \mu_{3}$ & 0 & $\mu_{1}$ & 0 & 0 & 0 & 0 & 0 \\
$\left(2,0, B_{3}(1)\right)$ & $\mu_{1}$ & 0 & $-\mu_{1}$ & 0 & 0 & 0 & 0 & 0 & 0 & 0 \\
$(1,2,0)$ & 0 & 0 & 0 & $-\mu_{1}-\mu_{2} \mu_{2}$ & 0 & 0 & 0 & $\mu_{1}$ & 0 \\
$(1,1,1)$ & $\mu_{3}$ & 0 & 0 & 0 & $-\mu_{1}-\mu_{2}-\mu_{3} \mu_{2}$ & $\mu_{1}$ & 0 & 0 & 0 \\
$(1,0,2)$ & 0 & $\mu_{3}$ & 0 & 0 & 0 & $-\mu_{1}-\mu_{3} 0$ & $\mu_{1}$ & 0 & 0 \\
$(0,2,1)$ & 0 & 0 & 0 & $\mu_{3}$ & 0 & 0 & $-\mu_{2}-\mu_{3} \mu_{2}$ & 0 & 0 \\
$(0,1,2)$ & 0 & 0 & 0 & 0 & $\mu_{3}$ & 0 & 0 & $-\mu_{2}-\mu_{3} 0$ & $\mu_{2}$ \\
$\left(B_{1}(1), 2,0\right)$ & 0 & 0 & 0 & 0 & 0 & 0 & $\mu_{2}$ & 0 & $-\mu_{2}$ & 0 \\
$\left(0, B_{2}(1), 2\right)$ & 0 & 0 & 0 & 0 & 0 & $\mu_{3}$ & 0 & 0 & 0 & $-\mu_{3}$ \\
\hline
\end{tabular}

columns in the middle correspond to the states and the transitions each associated with one interdeparture cycle. The inter-departure time starts by a transition from one of the states on the first column into one of the blue-colored states in the second column. The second inter-departure time terminates in one of the states on the right-hand side. The transitions associated with the other inter-departure cycles are drawn with dotted lines. Each transition from one of the states located in the second column of the figure to one of the states on the third column represent an increase in the number of parts produced on Station 1. Therefore, the inter-departure time distribution is the first passage time distribution from the states on the second column to the states on the third column. Furthermore, as discussed in Section 3, the steady-state probabilities of starting in one of the blue states in the second column is equal to the steady-state probabilities of absorption in one of the blue states in the third (and fourth) column.

The transitions that correspond to an increase in the number of parts produced on Station 1 are captured by the indicator matrix $G_{d}$. Table 3 gives the indicator matrix for the process depicted 
Figure 2 The state transition diagram of the process $\left\{X_{d}^{\prime}(t), t \geq 0\right\}$ associated with a closed three-station production line with exponential servers and 3 pallets

predecessor states first inter-departure time second inter-departure time exit states

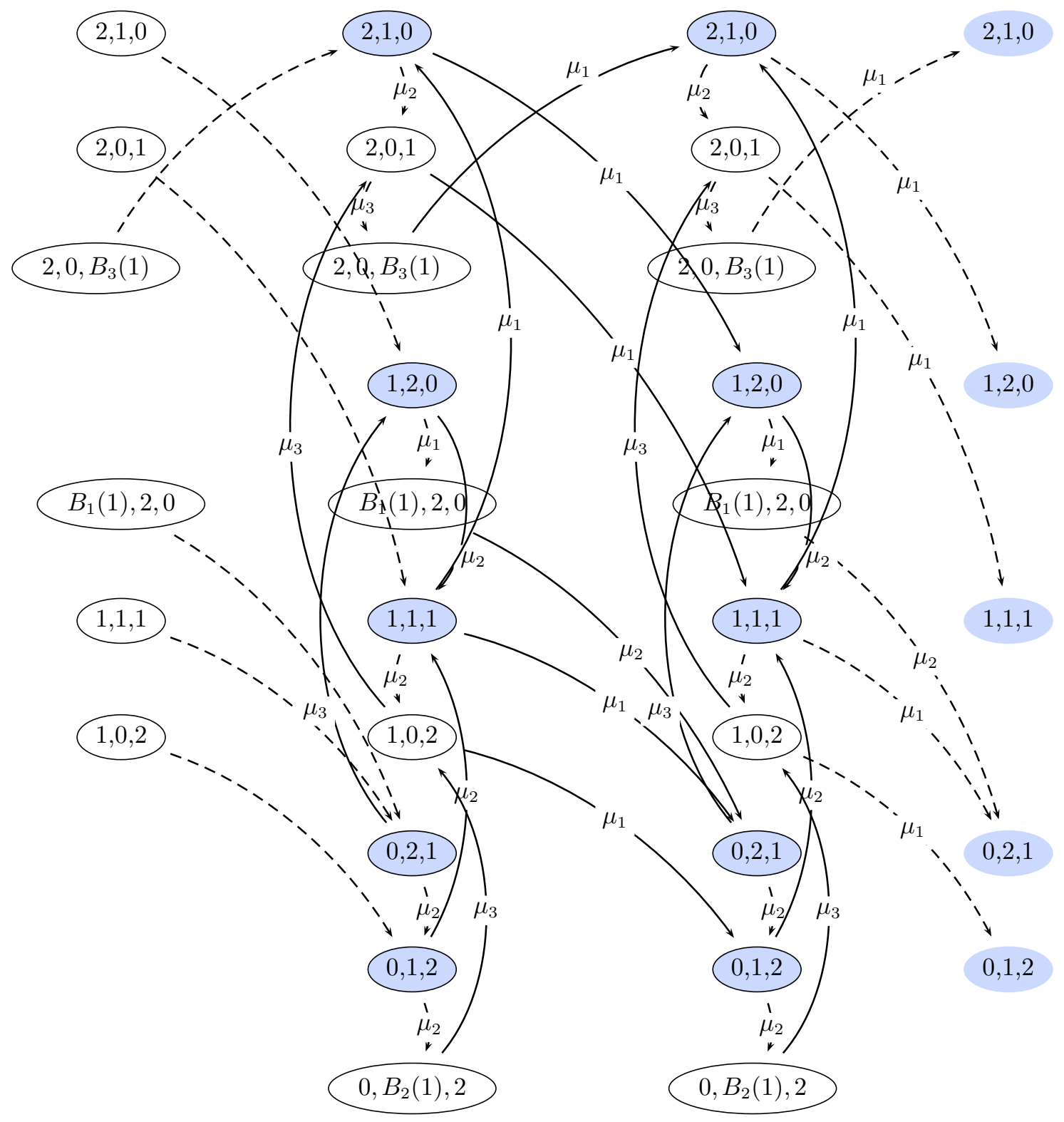

in Figure 2

Applying $Q$ and $G_{d}$ (given in Tables 2 and 3 to Equations (10) and (11), the transition rate matrices $Q_{d}$ and $R_{d}$ (given in Tables 4 and 5 ) are obtained.

Finally, since the matrices $Q_{d}$ and $R_{d}$ are defined, Equations(4) and (5) yield the steady-state entry probabilities and Equations (6) to (9) yield the distribution of the inter-departure time, its density, expectation and its variance, respectively. 
Table 3 The Indicator Matrix $G_{d}$ for the inter-departure time of a closed three-station production line with exponential servers and 3 pallets

\begin{tabular}{l|llllllllll} 
& $(2,1,0)$ & $(2,0,1)$ & $\left(2,0, B_{3}(1)\right.$ & $(1,2,0)$ & $(1,1,1)$ & $(1,0,2)$ & $(0,2,1)$ & $(0,1,2)$ & $\left(B_{1}(1), 2,0\right)$ & $\left(0, B_{2}(1), 2\right)$ \\
\hline$(2,1,0)$ & 0 & 0 & 0 & 1 & 0 & 0 & 0 & 0 & 0 & 0 \\
$(2,0,1)$ & 0 & 0 & 0 & 0 & 1 & 0 & 0 & 0 & 0 & 0 \\
$\left(2,0, B_{3}(1)\right)$ & 1 & 0 & 0 & 0 & 0 & 0 & 0 & 0 & 0 & 0 \\
$(1,2,0)$ & 0 & 0 & 0 & 0 & 0 & 0 & 0 & 0 & 0 & 0 \\
$(1,1,1)$ & 0 & 0 & 0 & 0 & 0 & 0 & 1 & 0 & 0 & 0 \\
$(1,0,2)$ & 0 & 0 & 0 & 0 & 0 & 0 & 0 & 1 & 0 & 0 \\
$(0,2,1)$ & 0 & 0 & 0 & 0 & 0 & 0 & 0 & 0 & 0 & 0 \\
$(0,1,2)$ & 0 & 0 & 0 & 0 & 0 & 0 & 0 & 0 & 0 & 0 \\
$\left(B_{1}(1), 2,0\right)$ & 0 & 0 & 0 & 0 & 0 & 0 & 1 & 0 & 0 & 0 \\
$\left(0, B_{2}(1), 2\right)$ & 0 & 0 & 0 & 0 & 0 & 0 & 0 & 0 & 0 & 0 \\
\hline
\end{tabular}

Table 4 Matrix $Q_{d}$ for inter-departure time of a closed three-station production line with exponential servers and 3 pallets

\begin{tabular}{l|llllllllll} 
& $(2,1,0)$ & $(2,0,1)$ & $\left(2,0, B_{3}(1)\right)$ & $(1,2,0)$ & $(1,1,1)$ & $(1,0,2)$ & $(0,2,1)$ & $(0,1,2)$ & $\left(B_{1}(1), 2,0\right)\left(0, B_{2}(1), 2\right)$ \\
\hline$(2,1,0)$ & $-\mu_{1}-\mu_{2} \mu_{2}$ & 0 & 0 & 0 & 0 & 0 & 0 & 0 & 0 \\
$(2,0,1)$ & 0 & $-\mu_{1}-\mu_{3} \mu_{3}$ & 0 & 0 & 0 & 0 & 0 & 0 & 0 \\
$\left(2,0, B_{3}(1)\right)$ & 0 & 0 & $-\mu_{1}$ & 0 & 0 & 0 & 0 & 0 & 0 & 0 \\
$(1,2,0)$ & 0 & 0 & 0 & $-\mu_{1}-\mu_{2} \mu_{2}$ & 0 & 0 & 0 & $\mu_{1}$ & 0 \\
$(1,1,1)$ & $\mu_{3}$ & 0 & 0 & 0 & $-\mu 1-\mu_{2}-\mu_{3} \mu_{2}$ & 0 & 0 & 0 & 0 \\
$(1,0,2)$ & 0 & $\mu_{3}$ & 0 & 0 & 0 & $-\mu_{1}-\mu_{3} 0$ & 0 & 0 & 0 \\
$(0,2,1)$ & 0 & 0 & 0 & $\mu_{3}$ & 0 & 0 & $-\mu_{2}-\mu_{3} \mu_{2}$ & 0 & 0 \\
$(0,1,2)$ & 0 & 0 & 0 & 0 & $\mu_{3}$ & 0 & 0 & $-\mu_{2}-\mu_{3} 0$ & $\mu_{2}$ \\
$\left(B_{1}(1), 2,0\right)$ & 0 & 0 & 0 & 0 & 0 & 0 & 0 & 0 & $-\mu_{2}$ & 0 \\
$\left(0, B_{2}(1), 2\right)$ & 0 & 0 & 0 & 0 & 0 & $\mu_{3}$ & 0 & 0 & 0 & $-\mu_{3}$ \\
\hline
\end{tabular}

Table 5 Matrix $R_{d}$ for inter-departure time of a closed three-station production line with exponential servers and 3 pallets

\begin{tabular}{l|llllllllll} 
& $(2,1,0)$ & $(2,0,1)$ & $\left(2,0, B_{3}(1)\right)$ & $(1,2,0)$ & $(1,1,1)$ & $(1,0,2)$ & $(0,2,1)$ & $(0,1,2)$ & $\left(B_{1}(1), 2,0\right)$ & $\left(0, B_{2}(1), 2\right)$ \\
\hline$(2,1,0)$ & 0 & 0 & 0 & $\mu_{1}$ & 0 & 0 & 0 & 0 & 0 & 0 \\
$(2,0,1)$ & 0 & 0 & 0 & 0 & $\mu_{1}$ & 0 & 0 & 0 & 0 & 0 \\
$\left(2,0, B_{3}(1)\right)$ & $\mu_{1}$ & 0 & 0 & 0 & 0 & 0 & 0 & 0 & 0 & 0 \\
$(1,2,0)$ & 0 & 0 & 0 & 0 & 0 & 0 & 0 & 0 & 0 & 0 \\
$(1,1,1)$ & 0 & 0 & 0 & 0 & 0 & 0 & $\mu_{1}$ & 0 & 0 & 0 \\
$(1,0,2)$ & 0 & 0 & 0 & 0 & 0 & 0 & 0 & $\mu_{1}$ & 0 & 0 \\
$(0,2,1)$ & 0 & 0 & 0 & 0 & 0 & 0 & 0 & 0 & 0 & 0 \\
$(0,1,2)$ & 0 & 0 & 0 & 0 & 0 & 0 & 0 & 0 & 0 & 0 \\
$\left(B_{1}(1), 2,0\right)$ & 0 & 0 & 0 & 0 & 0 & 0 & $\mu_{2}$ & 0 & 0 & 0 \\
$\left(0, B_{2}(1), 2\right)$ & 0 & 0 & 0 & 0 & 0 & 0 & 0 & 0 & 0 & 0 \\
\hline
\end{tabular}

Figure 3 shows the probability density function of the inter-departure time of Station 1 determined by using the methodology described above. While the inter-departure time distribution for a three-station line with exponential servers and infinite buffers in an open-loop configuration is exponential, operating the same system in a closed-loop configuration with finite buffers and with a given number of pallets circulating in the system causes blocking. As a result, the inter-departure time distribution is also non-exponential. 
Figure 3 Probability density function of the inter-departure time of a closed three-station production line with exponential servers and 3 pallets, $\mu_{1}=0.7, \mu_{2}=0.5, \mu_{3}=0.9$

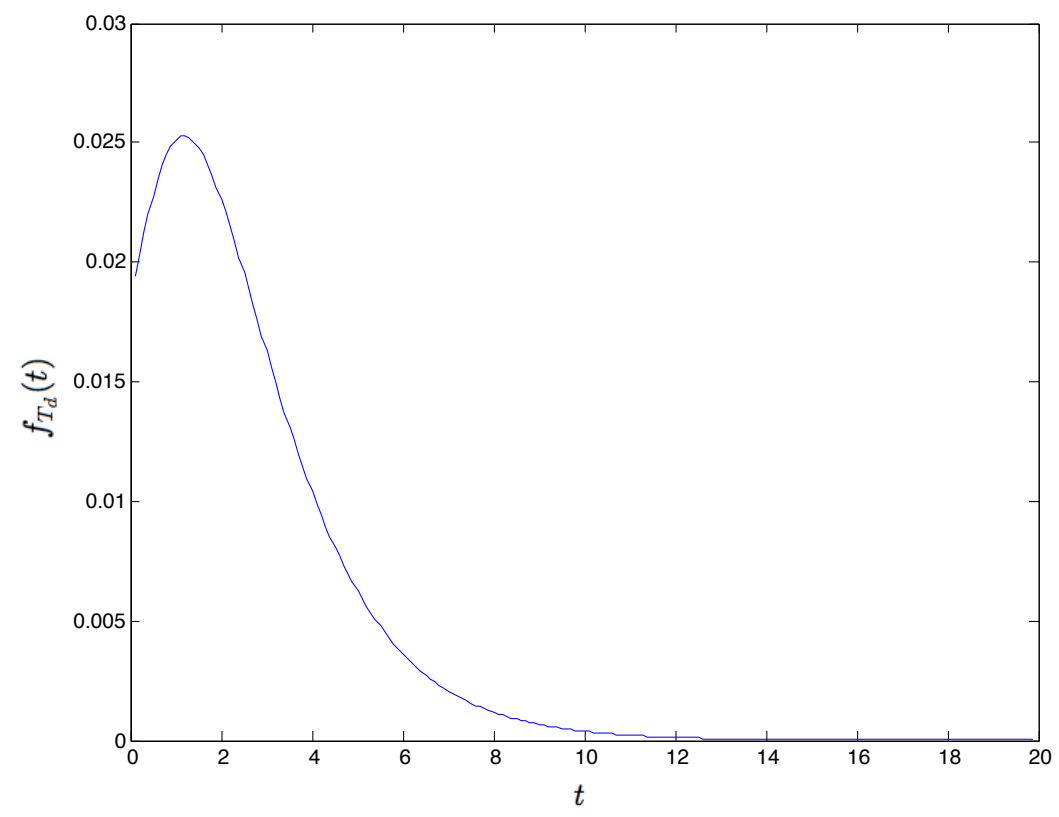

Table 6 The indicator matrix $G_{s}$ for the inter-start time a closed three-station production line with

exponential servers and 3 pallets

\begin{tabular}{l|llllllllll|} 
& $(2,1,0)$ & $(2,0,1)$ & $\left(2,0, B_{3}(1)\right)$ & $(1,2,0)$ & $(1,1,1)$ & $(1,0,2)$ & $(0,2,1)$ & $(0,1,2)$ & $\left(B_{1}(1), 2,0\right)\left(0, B_{2}(1), 2\right)$ \\
$(2,1,0)$ & 0 & 0 & 0 & 1 & 0 & 0 & 0 & 0 & 0 & 0 \\
$(2,0,1)$ & 0 & 0 & 0 & 0 & 1 & 0 & 0 & 0 & 0 & 0 \\
$\left(2,0, B_{3}(1)\right)$ & 1 & 0 & 0 & 0 & 0 & 0 & 0 & 0 & 0 & 0 \\
$(1,2,0)$ & 0 & 0 & 0 & 0 & 0 & 0 & 0 & 0 & 0 & 0 \\
$(1,1,1)$ & 0 & 0 & 0 & 0 & 0 & 0 & 0 & 0 & 0 & 0 \\
$(1,0,2)$ & 0 & 0 & 0 & 0 & 0 & 0 & 0 & 0 & 0 & 0 \\
$(0,2,1)$ & 0 & 0 & 0 & 1 & 0 & 0 & 0 & 0 & 0 & 0 \\
$(0,1,2)$ & 0 & 0 & 0 & 0 & 1 & 0 & 0 & 0 & 0 & 0 \\
$\left(B_{1}(1), 2,0\right)$ & 0 & 0 & 0 & 0 & 0 & 0 & 0 & 0 & 0 & 0 \\
$\left(0, B_{2}(1), 2\right)$ & 0 & 0 & 0 & 0 & 0 & 1 & 0 & 0 & 0 & 0
\end{tabular}

\subsection{Inter-start Time Distribution}

As in the previous case, we illustrate the inter-start time distribution by a state transition diagram of the process that captures the processing start of parts on Station 1 for two succeeding inter-start time cycles, see Figure 4.

In order to implement the first passage time analysis for the inter-start time distribution, it is necessary to define the indicator matrix $G_{s}$. The transitions that correspond to an increase in the number of parts that have started the operation on Station 1 are given in the indicator matrix $G_{s}$ as shown in Table 6.

Once $G_{s}$ is defined, $Q_{s}$ and $R_{s}$ are obtained by using Equations 12 and (13) and, therefore, the inter-start time distribution and density can be determined directly from Equations (6) and 
Figure 4 The state transition diagram of the process $\left\{X_{s}^{\prime}(t), t \geq 0\right\}$ associated with a closed three-station production line with exponential servers and 3 pallets

predecessor states first inter-start time $\quad$ second inter-start time $\quad$ exit states

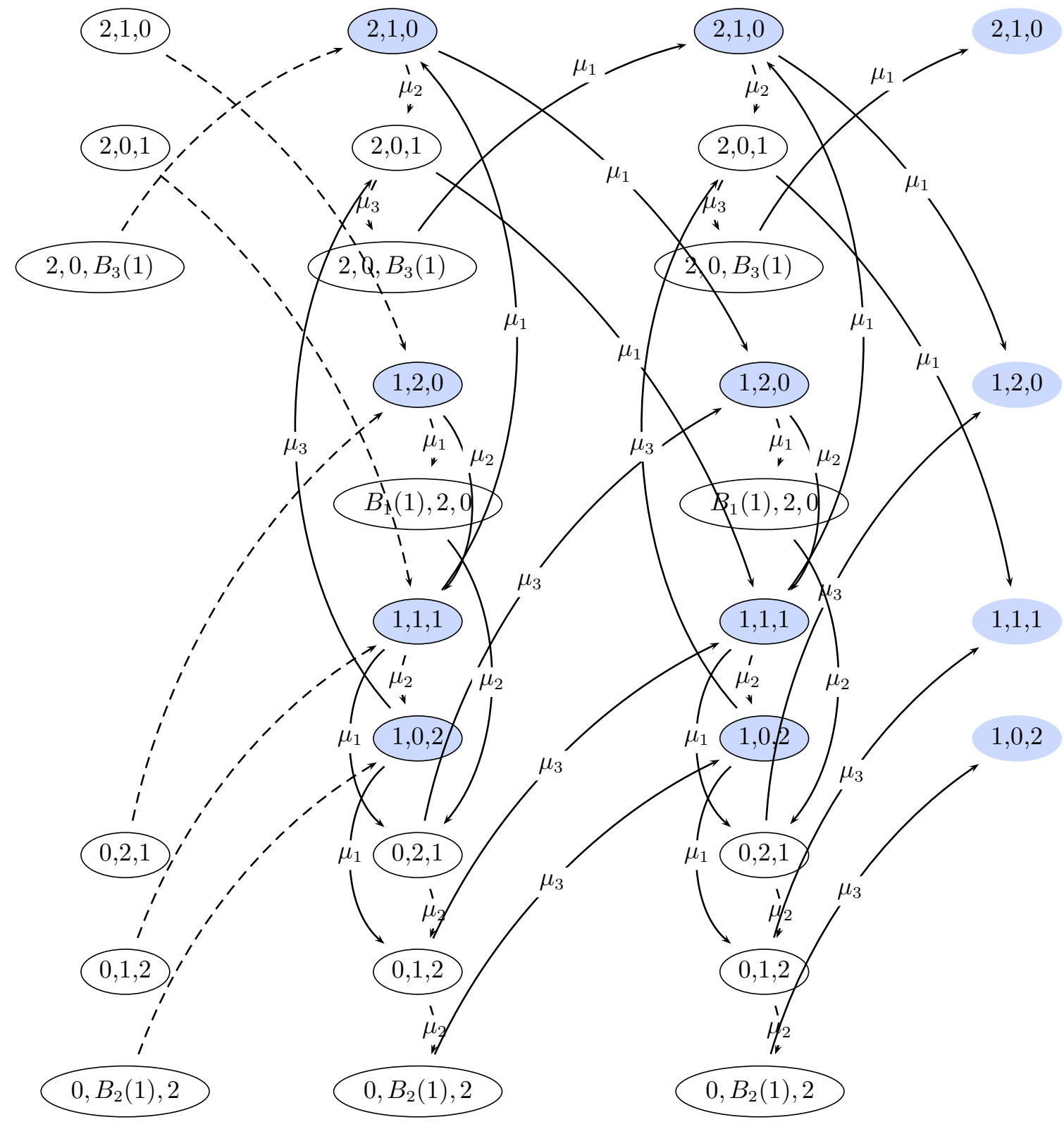

Equations (7).

Figure 5 shows the probability density function of the inter-start time distribution determined by using the methodology described above. The shape of the inter-start time distribution is similar to the shape of the inter-departure time distribution of the same system depicted in Figure 3 but the inter-start and inter-departure time distributions are not identical. 
Table 7 Matrix $R_{s}$ for inter-start time of a closed three-station production line with exponential servers and 3 pallets

\begin{tabular}{l|llllllllll|} 
& $(2,1,0)$ & $(2,0,1)$ & $\left(2,0, B_{3}(1)\right.$ & $(1,2,0)$ & $(1,1,1)$ & $(1,0,2)$ & $(0,2,1)$ & $(0,1,2)$ & $\left(B_{1}(1), 2,0\right)$ & $\left(0, B_{2}(1), 2\right)$ \\
\hline$(2,1,0)$ & 0 & 0 & 0 & $\mu_{1}$ & 0 & 0 & 0 & 0 & 0 & 0 \\
$(2,0,1)$ & 0 & 0 & 0 & 0 & $\mu_{1}$ & 0 & 0 & 0 & 0 & 0 \\
$\left(2,0, B_{3}(1)\right)$ & $\mu_{1}$ & 0 & 0 & 0 & 0 & 0 & 0 & 0 & 0 & 0 \\
$(1,2,0)$ & 0 & 0 & 0 & 0 & 0 & 0 & 0 & 0 & 0 & 0 \\
$(1,1,1)$ & 0 & 0 & 0 & 0 & 0 & 0 & 0 & 0 & 0 & 0 \\
$(1,0,2)$ & 0 & 0 & 0 & 0 & 0 & 0 & 0 & 0 & 0 & 0 \\
$(0,2,1)$ & 0 & 0 & 0 & $\mu_{3}$ & 0 & 0 & 0 & 0 & 0 & 0 \\
$(0,1,2)$ & 0 & 0 & 0 & 0 & $\mu_{3}$ & 0 & 0 & 0 & 0 & 0 \\
$\left(B_{1}(1), 2,0\right)$ & 0 & 0 & 0 & 0 & 0 & 0 & 0 & 0 & 0 & 0 \\
$\left(0, B_{2}(1), 2\right)$ & 0 & 0 & 0 & 0 & 0 & $\mu_{3}$ & 0 & 0 & 0 & 0 \\
\hline
\end{tabular}

Table 8 Matrix $Q_{s}$ for inter-start time of a closed three-station production line with exponential servers and 3

\begin{tabular}{|c|c|c|c|c|c|c|c|c|c|c|}
\hline & & & & & pallets & & & & & \\
\hline & $(2,1,0)$ & $(2,0,1)$ & $\left(2,0, B_{3}(1)\right)$ & $(1,2,0)$ & $(1,1,1)$ & $(1,0,2)$ & $(0,2,1)$ & $(0,1,2)$ & $\left(B_{1}(1), 2,0\right)$ & $\left(0, B_{2}(1), 2\right)$ \\
\hline$(2,1,0)$ & $-\mu_{1}-\mu_{2}$ & $\mu_{2}$ & 0 & 0 & 0 & 0 & 0 & 0 & 0 & 0 \\
\hline$(2,0,1)$ & 0 & $-\mu_{1}-\mu_{3}$ & $\mu_{3}$ & 0 & 0 & 0 & 0 & 0 & 0 & 0 \\
\hline$\left(2,0, B_{3}(1)\right)$ & 0 & 0 & $-\mu_{1}$ & 0 & 0 & 0 & 0 & 0 & 0 & 0 \\
\hline$(1,2,0)$ & 0 & 0 & 0 & $-\mu_{1}-\mu_{2}$ & $\mu_{2}$ & 0 & 0 & 0 & $\mu_{1}$ & 0 \\
\hline$(1,1,1)$ & $\mu_{3}$ & 0 & 0 & 0 & $-\mu_{1}-\mu_{2}-\mu_{3}$ & $\mu_{2}$ & $\mu_{1}$ & 0 & 0 & 0 \\
\hline$(1,0,2)$ & 0 & $\mu_{3}$ & 0 & 0 & 0 & $-\mu_{1}-\mu_{3}$ & & $\mu_{1}$ & 0 & 0 \\
\hline$(0,2,1)$ & 0 & 0 & 0 & 0 & 0 & 0 & $-\mu_{2}-\mu_{3}$ & $\mu_{2}$ & 0 & 0 \\
\hline$(0,1,2)$ & 0 & 0 & 0 & 0 & 0 & 0 & 0 & $-\mu_{2}-\mu_{3}$ & 0 & $\mu_{2}$ \\
\hline$\left(B_{1}(1), 2,0\right)$ & 0 & 0 & 0 & 0 & 0 & 0 & $\mu_{2}$ & 0 & $-\mu_{2}$ & 0 \\
\hline$\left(0, B_{2}(1), 2\right)$ & 0 & 0 & 0 & 0 & 0 & 0 & 0 & 0 & 0 & $-\mu_{3}$ \\
\hline
\end{tabular}

Figure 5 Probability density function of the inter-start time of a closed three-station production line with exponential servers and 3 pallets, $\mu_{1}=0.7, \mu_{2}=0.5, \mu_{3}=0.9$

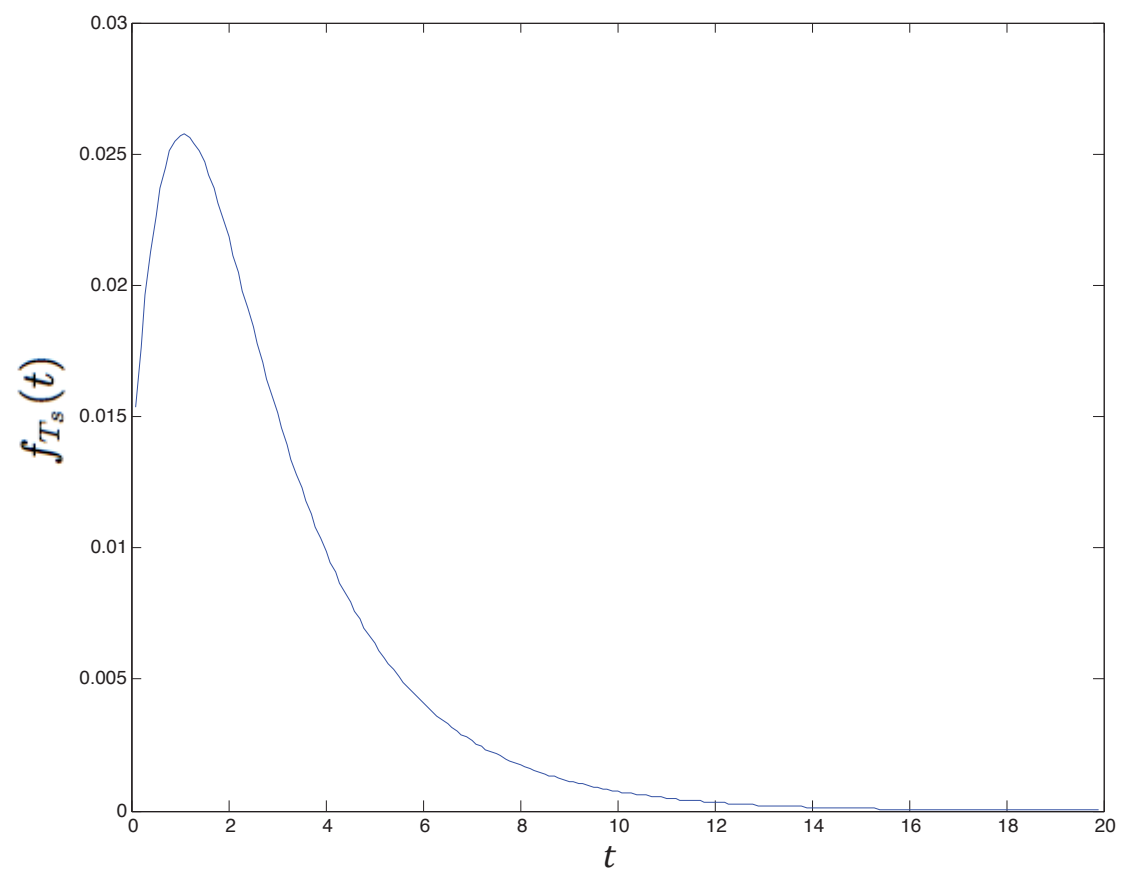


Table 9 The Indicator Matrix $G_{c}$ for the cycle time of a closed three-station production line with exponential servers and 3 pallets

\begin{tabular}{l|llllllllll} 
& $(2,1,0)$ & $(2,0,1)$ & $\left(2,0, B_{3}(1)\right.$ & $(1,2,0)$ & $(1,1,1)$ & $(1,0,2)$ & $(0,2,1)$ & $(0,1,2)$ & $\left(B_{1}(1), 2,0\right)$ & $\left(0, B_{2}(1), 2\right)$ \\
\hline$(2,1,0)$ & 0 & 0 & 0 & 0 & 0 & 0 & 0 & 0 & 0 & 0 \\
$(2,0,1)$ & 0 & 0 & 0 & 0 & 0 & 0 & 0 & 0 & 0 & 0 \\
$\left(2,0, B_{3}(1)\right)$ & 1 & 0 & 0 & 0 & 0 & 0 & 0 & 0 & 0 & 0 \\
$(1,2,0)$ & 0 & 0 & 0 & 0 & 0 & 0 & 0 & 0 & 0 & 0 \\
$(1,1,1)$ & 1 & 0 & 0 & 0 & 0 & 0 & 0 & 0 & 0 & 0 \\
$(1,0,2)$ & 0 & 1 & 0 & 0 & 0 & 0 & 0 & 0 & 0 & 0 \\
$(0,2,1)$ & 0 & 0 & 0 & 1 & 0 & 0 & 0 & 0 & 0 & 0 \\
$(0,1,2)$ & 0 & 0 & 0 & 0 & 1 & 0 & 0 & 0 & 0 & 0 \\
$\left(B_{1}(1), 2,0\right)$ & 0 & 0 & 0 & 0 & 0 & 0 & 0 & 0 & 0 & 0 \\
$\left(0, B_{2}(1), 2\right)$ & 0 & 0 & 0 & 0 & 0 & 1 & 0 & 0 & 0 & 0 \\
\hline
\end{tabular}

\subsection{Cycle Time Distribution}

In order to analyze the cycle time, the state space is extended to track the location of each part on each station from the initial state where the first part is located in front of the input buffer of Station 1 to the state where the same part finishes its operation and departs from Station 3. Figure 6 depicts the extended state transition diagram for the cycle time distribution. The state transition rate matrix for the extended process is in the form given in Equation 19.

$$
Q_{c}^{\prime}=\left[\begin{array}{ccc|ccc}
\tilde{Q}_{c} & \tilde{R}_{c} & 0 & 0 & 0 & 0 \\
0 & \tilde{Q}_{c} & \tilde{R}_{c} & 0 & 0 & 0 \\
0 & 0 & \tilde{Q}_{c} & \tilde{R}_{c} & 0 & 0 \\
\hline 0 & 0 & 0 & 0 & 0 & 0
\end{array}\right]
$$

where $\tilde{Q}_{c}$ is a $10 \times 10$ infinitesimal generator submatrix for the transitions from the states in $S_{m}$ to the states in $S_{m}$ and $\tilde{R}_{c}$ is a $10 \times 10$ submatrix with elements that are the transition rates from the states in $S_{m}$ to the states $S_{m-1}, m=1,2,3$.

The transitions that correspond to a decrease in the work-in-process through departures from Station 3 are identified by the indicator matrix $G_{c}$. Table 9 gives the indicator matrix for the cycle time.

By using Equations $(16)$ and $(17)$, the matrices $\tilde{Q}_{c}$ and $\tilde{R}_{c}$ are constructed as given in Tables 10 and 11 .

As a result, the cycle time distribution and its density are completely determined by Equations (6) and (7). Figure 7 depicts the probability density function of the cycle time for the example of a closed 3-station production line with exponential servers and 3 pallets.

\section{Numerical Experiments}

In this section, we give results of exploratory numerical experiments that show how the methodology presented in this study can be used to analyze different phenomena regarding the variability 
Figure 6 State Transition Diagram of $\left\{X_{c}^{\prime}(t), t \geq 0\right\}$ associated with a closed three-station production line with exponential servers and 3 pallets

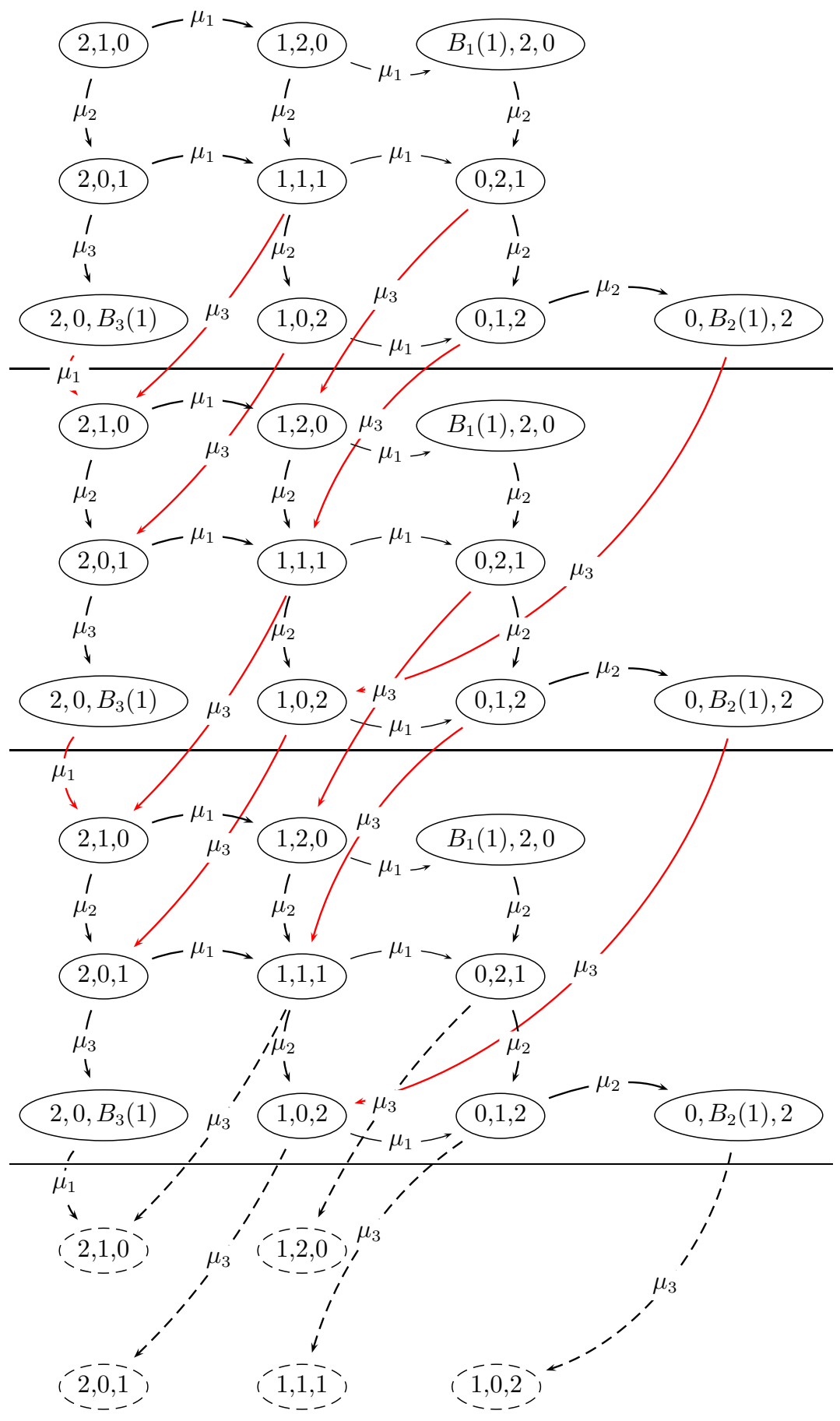

of flow and the output in production systems.

In the first part, we show how the processing rate, the coefficient of variation of the processing time, and buffer capacities affect the coefficient of variation and the distribution of the interdeparture time. In the second part, we provide numerical results of selected queuing networks. These results are especially of interest for comparisons with the results of approximate methods. 
Table 10 Transition rate matrix $\tilde{Q}_{c}$ for a closed three-station production line with exponential servers and 3

\begin{tabular}{|c|c|c|c|c|c|c|c|c|c|c|}
\hline & & & & & pallets & & & & & \\
\hline & $(2,1,0)$ & $(2,0,1)$ & $\left(2,0, B_{3}(1)\right)$ & $(1,2,0)$ & $(1,1,1)$ & $(1,0,2)$ & $(0,2,1)$ & $(0,1,2)$ & $\left(B_{1}(1), 2,0\right)$ & $\left(0, B_{2}(1), 2\right)$ \\
\hline$\overline{(2,1,0)}$ & $-\mu_{1}-\mu_{2}$ & & 0 & $\mu_{1}$ & 0 & 0 & 0 & 0 & 0 & 0 \\
\hline$(2,0,1)$ & 0 & $-\mu_{1}-\mu_{3}$ & $\mu_{3}$ & 0 & $\mu_{1}$ & 0 & 0 & 0 & 0 & 0 \\
\hline$\left(2,0, B_{3}(1)\right)$ & 0 & 0 & $-\mu_{1}$ & 0 & 0 & 0 & 0 & 0 & 0 & 0 \\
\hline$(1,2,0)$ & 0 & 0 & 0 & $-\mu_{1}-\mu_{2}$ & $\mu_{2}$ & 0 & 0 & 0 & $\mu_{1}$ & 0 \\
\hline$(1,1,1)$ & 0 & 0 & 0 & 0 & $-\mu_{1}-\mu_{2}-\mu_{3}$ & $\mu_{2}$ & $\mu_{1}$ & 0 & 0 & 0 \\
\hline$(1,0,2)$ & 0 & 0 & 0 & 0 & 0 & $-\mu_{1}-\mu_{3}$ & 0 & $\mu_{1}$ & 0 & 0 \\
\hline$(0,2,1)$ & 0 & 0 & 0 & $\mu_{3}$ & 0 & 0 & $-\mu_{2}-\mu_{3}$ & $\mu_{2}$ & 0 & 0 \\
\hline$(0,1,2)$ & 0 & 0 & 0 & 0 & 0 & 0 & 0 & $-\mu_{2}-\mu_{3}$ & 0 & $\mu_{2}$ \\
\hline$\left(B_{1}(1), 2,0\right)$ & 0 & 0 & 0 & 0 & 0 & 0 & $\mu_{2}$ & 0 & $-\mu_{2}$ & 0 \\
\hline$\left(0, B_{2}(1), 2\right)$ & 0 & 0 & 0 & 0 & 0 & $\mu_{3}$ & 0 & 0 & 0 & $-\mu_{3}$ \\
\hline
\end{tabular}

Table 11 Transition rate matrix $\tilde{R}_{c}$ for a closed three-station production line with exponential servers and 3

\begin{tabular}{|c|c|c|c|c|c|c|c|c|c|c|}
\hline \multicolumn{11}{|c|}{ pallets } \\
\hline & $(2,1,0)$ & $(2,0,1)$ & $\left(2,0, B_{3}(1)\right)$ & $(1,2,0)$ & $(1,1,1)$ & $(1,0,2)$ & $(0,2,1)$ & $(0,1,2)$ & $\left(B_{1}(1), 2,0\right)$ & $\left(0, B_{2}(1), 2\right)$ \\
\hline$(2,1,0)$ & 0 & 0 & 0 & 0 & 0 & 0 & 0 & 0 & 0 & 0 \\
\hline$(2,0,1)$ & 0 & 0 & 0 & 0 & 0 & 0 & 0 & 0 & 0 & 0 \\
\hline$\left(2,0, B_{3}(1)\right)$ & $\mu_{1}$ & 0 & 0 & 0 & 0 & 0 & 0 & 0 & 0 & 0 \\
\hline$(1,2,0)$ & 0 & 0 & 0 & 0 & 0 & 0 & 0 & 0 & 0 & 0 \\
\hline$(1,1,1)$ & $\mu_{3}$ & 0 & 0 & 0 & 0 & 0 & 0 & 0 & 0 & 0 \\
\hline$(1,0,2)$ & 0 & $\mu_{3}$ & 0 & 0 & 0 & 0 & 0 & 0 & 0 & 0 \\
\hline$(0,2,1)$ & 0 & 0 & 0 & $\mu_{3}$ & 0 & 0 & 0 & 0 & 0 & 0 \\
\hline$(0,1,2)$ & 0 & 0 & 0 & 0 & $\mu_{3}$ & 0 & 0 & 0 & 0 & 0 \\
\hline$\left(B_{1}(1), 2,0\right)$ & 0 & 0 & 0 & 0 & 0 & 0 & 0 & 0 & 0 & 0 \\
\hline$\left(0, B_{2}(1), 2\right)$ & 0 & 0 & 0 & 0 & 0 & $\mu_{3}$ & 0 & 0 & 0 & 0 \\
\hline
\end{tabular}

Figure 7 Probability Density Function of the cycle time of Station 1 of a closed three-station production line with exponential servers and 3 pallets, $\mu_{1}=0.7, \mu_{2}=0.5, \mu_{3}=0.9$

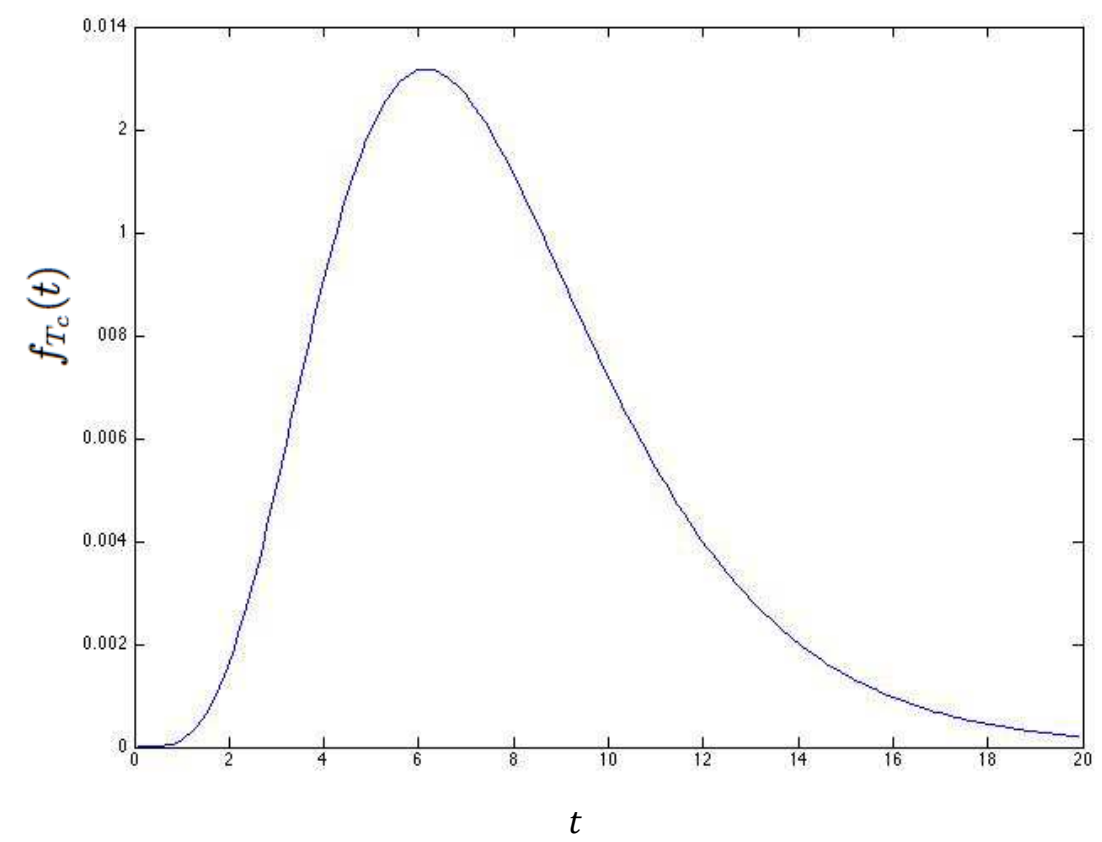

These examples show that the proposed method allows the analysis of queueing networks with different configurations and obtain various performance measures related to the variability of the output and flow. 
Figure 8 Coefficient of variation of the inter-departure Time of Station 1 of a closed three-station production line with exponential servers and 3 pallets, $\mu_{2}=1, \mu_{3}=1$

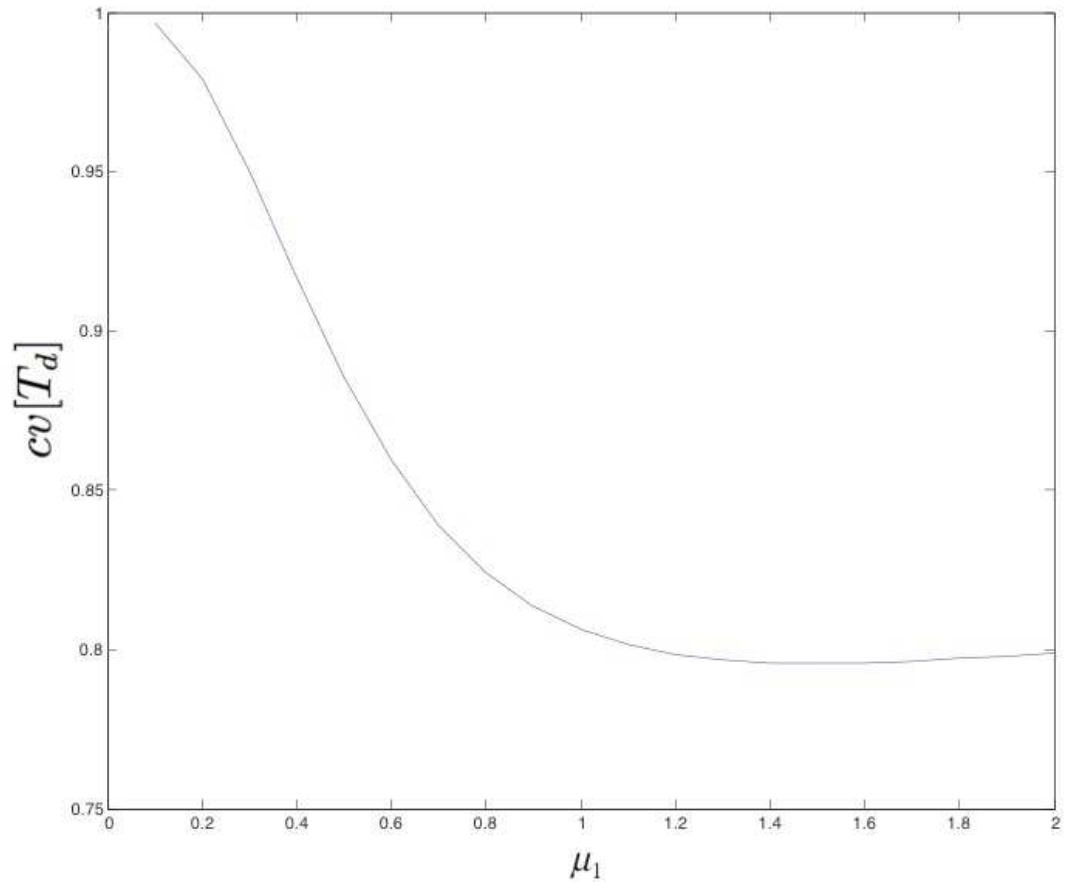

\subsection{Effects of the processing time and the buffer capacities on the variability of the inter-departure time}

We first investigate how the variability of flow is affected depending on the system parameters.

\subsubsection{Effect of the Processing Rate on the Variability of the Inter-departure Time}

Figure 8 shows the coefficient of variation of the inter-departure time $c v\left[T_{d}\right]$ depending on the processing rate of Station 1 of a three-station production line with exponential servers and blocking. When the processing rate of Station 1 is lower than the other two stations, increasing the processing rate of Station 1 decreases the coefficient of variation of the inter-departure time. However, as the processing time of Station 1 increases and exceeds the processing rates of the other two stations, the coefficient of variation starts to increase slightly.

\subsubsection{Effect of the Service Time Distribution on the Flow Variability}

In order to study the effects of the service time distribution, we use a production line with machines that have phase-type service distributions. The state space of this system, the transition matrix, and the indicator matrices are constructed automatically by using the approach described in Lagershausen (2012).

State Space Model. We first give the state space model for a production line with $K$ phasetype servers and $M$ pallets circulating in the system. Station $k$ has a finite buffer capacity of $b_{k}$ and its service time has $h_{k}$ phases. In the state space of the queueing model of this system, a state $s$ in the state space $S$ is made up of the queue length $q_{k}$ and the phase value $p h_{k}$, each for every 
station $k=1, \ldots, K$. A state $s$ is described by

$$
s=\left\{\left[q_{1}, p h_{1}\right],\left[q_{2}, p h_{2}\right], \ldots,\left[q_{K}, p h_{K}\right]\right\}
$$

under the restrictions

$$
\begin{aligned}
& M=\sum_{k=1}^{K} n_{k} \quad \text { with } n_{k} \leq\left(b_{k}+1\right) \forall k \\
& q_{k}=\left\{\begin{array}{ll}
0 & \text { if } n_{k}=0 \\
n_{k}-1 & \text { if } n_{k}>0
\end{array} \quad \forall i\right. \\
& p h_{k}=\left\{\begin{array}{ll}
0 & \text { if } n_{k}=0 \\
\left\{1, \ldots, h_{k}\right\} & \text { if } n_{k}>0 \text { and } n_{k+1}<b_{k+1}+1 \\
\left\{1, \ldots, h_{k}, B_{k}\right\} & \text { if } n_{k}>0 \text { and } n_{k+1}=b_{k+1}+1 .
\end{array} \quad \forall i\right.
\end{aligned}
$$

Equation (21) shows that the state space $S$ is restricted in the way that the sum of customers over all stations $k$, denoted by $n_{k}$, must be equal to the required number of customers $M$. Thereby, at each station $k, n_{k}$ must not exceed the station capacity $\left(b_{k}+1\right)$. The queue length, $q_{k}$, equals $q_{k}=\max \left\{n_{k}-1,0\right\}$, as indicated in Equation (22). Equation (23) shows that the range of values for the phase value $p h_{k}$ depends on $n_{k}$ as well. If station $k$ is idle $\left(n_{k}=0\right)$, the phase value $p h_{k}$ equals $p h_{k}=0$. If Station $k$ is active, the phase value $p h_{k}$ indicates in which phase of the phasetype distribution the process is active $\left(p h_{k}=\left\{1, \ldots, h_{k}\right\}\right)$. Station $k$ can be active if there is at least one customer at station $k\left(n_{k}>0\right)$ and if that station $k$ is not blocked. If (i) a customer at Station $k$ is in service $\left(n_{k}>0\right)$ and (ii) the succeeding buffer is full $\left(n_{k+1}=b_{k+1}+1\right)$, station $k$ is either active in any phase $p h_{k}=\left\{1, \ldots, h_{k}\right\}$ or blocked, $p h_{k}=B_{k}$. Since we assume blocking-after-service, blocking occurs if the last phase is finished and the succeeding buffer is full.

By using this model, we can represent service time distributions with any coefficient of variation by using the minimum number of phases as described in Lagershausen (2012).

Figure 9 illustrates the probability density functions of the inter-departure time of Station 1 of a three-station system with different coefficient of variation of the processing time distribution, a buffer capacity of 2 at all stations, $b_{k}=2, k=1,2,3$, and the processing rates $\mu_{1}=3, \mu_{2}=2$, $\mu_{3}=2$.

\subsubsection{Effect of the Buffer Capacity on the Variability of the Inter-departure Time}

Figure 10 depicts the probability density functions of the inter-departure time of Station 1 of a four-station system with different buffer capacities and the processing rate $\mu_{k}=1, k=1,2,3,4$ and a coefficient of variation of $c_{k}^{2}=2, k=1,2,3,4$. The graphic shows that the variability of the inter-departure time decreases with higher buffer capacity.

\subsection{Computational Results}

5.2.1. Effects of the Number of Stations, Number of Parts and Buffer Capacities on the State Space for Exponential Servers In this section, we examine how the number 
Figure 9 Probability density function of the inter-departure time of Station 1 of a closed three-station system with different coefficient of variations of the processing time, $\mu_{1}=3, \mu_{2}=2, \mu_{3}=2, b_{i}=2 \forall i$

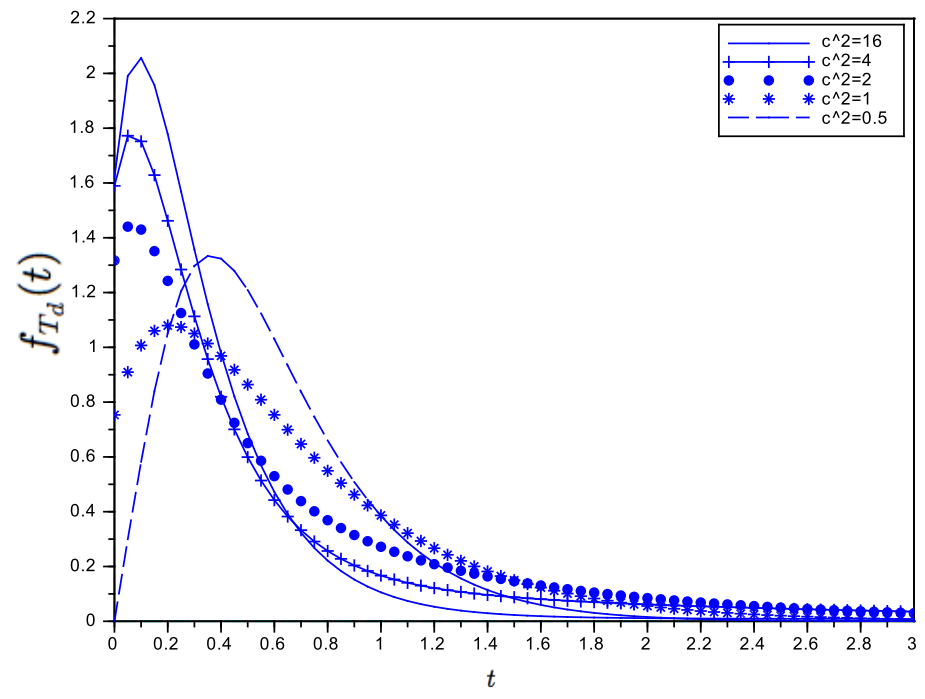

Figure 10 Probability density function of the inter-departure time of Station 1 of a four-station system with different buffer capacities, $\mu_{i}=1 \forall i, c_{i}^{2}=2 \forall i$

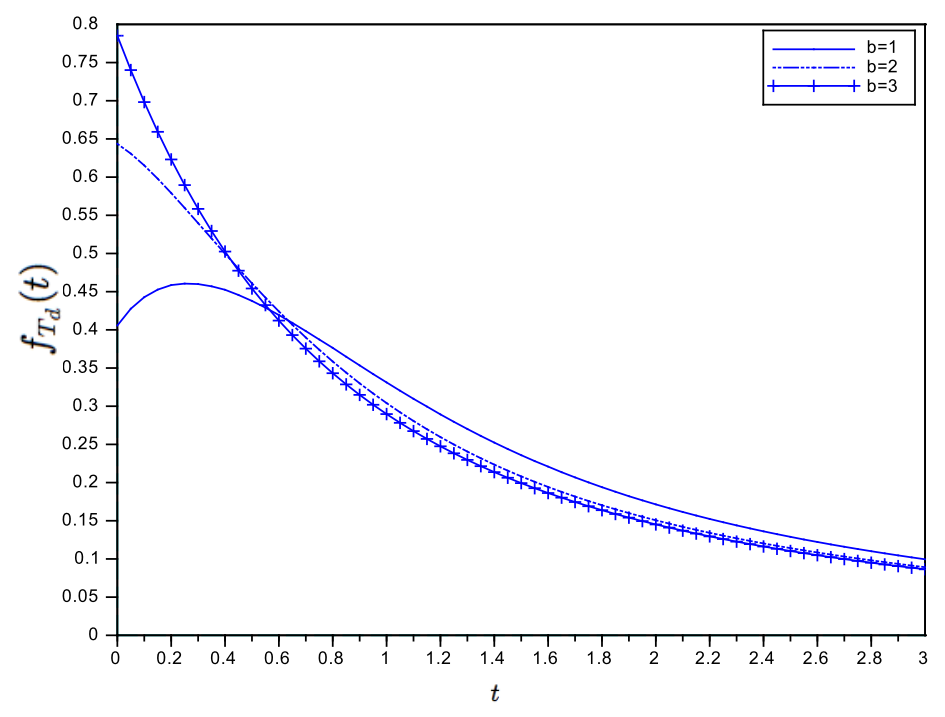

of states in the state space increases with the number of stations, the number of parts, and with the buffer capacity in the case of exponential servers. Since the method proposed in this study is state-space based, its computational performance is affected by a rapid growth of the state space which depends on the system parameters. Note that this is a limitation of all state-space based methods and that it is not specific to our method.

In general, the number of states in the state space of a closed queueing network with $K$ stations with exponential servers, with identical buffer capacity of $b$ at each station, with one server at each station, and $M$ circulating parts can be derived by using the rules of counting the number of ways $M$ indistinguishable balls can be allocated to $K$ distinguishable containers. If we ignore 
the buffer capacities, the number of ways $M$ parts can be distributed to $K$ stations is

$$
\left(\begin{array}{c}
M+K-1 \\
K-1
\end{array}\right)=\frac{(M+K-1) !}{M !(K-1) !} .
$$

Now in order to determine the number of ways $M$ parts can be distributed to $K$ stations with the restriction that the number of parts at one particular station, say at station $i$, is less than or equal to $b+1$ ( $b$ parts in the buffer and one part on the server), we first analyze the complement case. That is, we determine the number of ways $M$ parts can be distributed to $K$ stations with $b+2$ parts allocated to station $i$; and the remaining $M-(b+2)$ parts are distributed to $K$ stations. Following the result given above, this is

$$
\left(\begin{array}{c}
M-b+K-3 \\
K-1
\end{array}\right)
$$

Let us first consider the case where $b+1 \leq M<2(b+1)$. In this case, at most one station can have $b+1$ parts in a given state. Since this station can be selected in $K$ different ways, the number of ways $M$ parts can be distributed to $K$ stations with the restriction that the number of parts at each station is less than or equal to $b+1$ can be written as

$$
\left(\begin{array}{c}
M+K-1 \\
K-1
\end{array}\right)-K\left(\begin{array}{c}
M-b+K-3 \\
K-1
\end{array}\right)
$$

For the general case, there can be at most $\left\lfloor\frac{M}{b+1}\right\rfloor$ stations with $b+1$ parts in the state space. Therefore, the number of ways $M$ parts can be distributed to $K$ stations with the restriction that each station can have at most $b+1$ parts, denoted with $N(M, K, b)$ can be written as

$$
N(M, K, b)=\left(\begin{array}{c}
M+K-1 \\
K-1
\end{array}\right)+\sum_{n=1}^{\left\lfloor\frac{M}{b+1}\right\rfloor}(-1)^{n}\left(\begin{array}{c}
K \\
n
\end{array}\right)\left(\begin{array}{c}
M-(b+2) n+K-1 \\
K-1
\end{array}\right)
$$

In the state space, there are additional states that indicate particular stations are blocked since the following station has $b+1$ parts. Each state where there are $b+1$ parts at a station and there is at least one part in the preceding station yields an additional blocked state.

The number of states where a given station has exactly $b+1$ parts is equal to the number of ways $M-(b+1)$ parts are distributed to the remaining $K-1$ stations. This can be written as $N(M-b-1, K-1, b)$ where $N(M, K, b)$ is the function that gives the number of ways $M$ parts can be distributed to $K$ stations with at most $b+1$ parts at each station given in Equation (24). Similarly, the number of states where a given station has exactly $b+1$ parts and the preceding station has exactly 0 parts is equal to the number of ways $M-(b+1)$ parts are distributed to the remaining $K-2$ stations, $N(M-b-1, K-2, b)$ when $K \geq 3$.

Let us again consider the case where $b+1 \leq M<2(b+1)$ where at most one station can have 
$b+1$ parts in a given state. In this case, the number of additional states corresponding to the blocked states is

$$
K(N(M-b-1, K-1, b)-N(M-b-1, K-2, b))
$$

In addition, states where there are $b+1$ parts at a station and there is at least one part in the preceding station and other stations are blocked also yield a blocked state. In a given state, in addition to the station with $b+1$ parts and its preceding station, there can be at most $\left\lfloor\frac{M}{b+1}\right\rfloor-1$ blocked stations. Therefore, the number of additional states that represent blocking for the general case is denoted with $N_{B}(M, K, b)$ and given as

$N_{B}(M, K, b)=K N(M-b-1, K-1, b)-K \sum_{n=0}^{\left\lfloor\frac{M}{b+1}\right\rfloor-1}\left(\begin{array}{c}K-2 \\ n\end{array}\right) N(M-(n+1)(b+1), K-2-n, b)$.

Finally, the total number of states in the state space to represent a closed queueing network with $K$ exponential states with buffer capacities of $b$ and $M$ circulating pallets is

$$
N_{T}(M, K, b)=N(M, K, b)+N_{B}(M, K, b)
$$

where the functions $N(M, K, b)$ is given in Equation (24) and $N_{B}(M, K, b)$ are defined in Equation (25).

This analysis shows that the number of states in the state space is polynomial in $M$ and in $b$ and exponential in $K$. The computational complexity of the proposed method that includes matrix inversions and multiplications is polynomial. Therefore, the method we propose will be limited by the number of stations. As the number of stations increases, the computational time will increase exponentially and will limit the applicability of the method to analyze larger systems. As mentioned above, the method is proposed to obtain exact distribution of the inter-event times that cannot be obtained by other methods. These results will be useful to compare the accuracy of approximation methods that are developed to analyze larger systems.

5.2.2. Computational Results and Performance In the following part, we provide results of a number of numerical experiments and investigate the computational performance of the system.

Figure 11 shows the expected value of the inter-start time of Station 1 of a three-station exponential closed system with a buffer capacity of 10 at each station and the following processing rates: $\mu_{1}=0.7, \mu_{2}=0.5, \mu_{3}=0,9$.

Tables 12, 13 and 14 report the expected value and the variance of the inter-start and interdeparture time distribution at each station for a two-station system with different distributions, a three-station exponential CQN, and a Coxian 4-station 5-buffer system. 
Figure 11 Expected value of the inter-start time of Station 1 of a three-station system $b_{i}=10 \forall i, \mu_{1}=0.7, \mu_{2}=$ $0.5, \mu_{3}=0.9, c_{i}^{2}=1 \forall i$

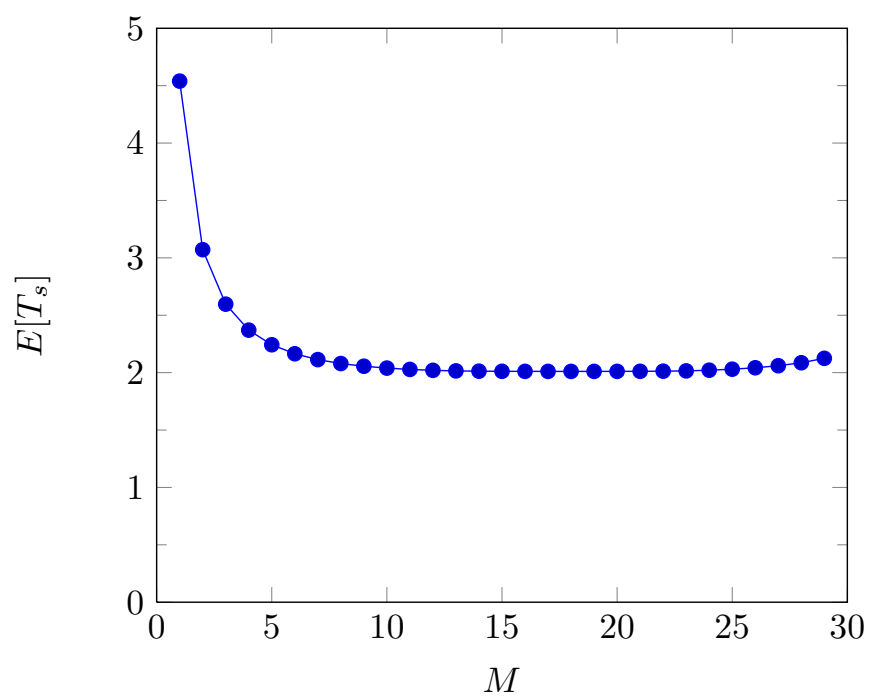

Table 12 Computational Results a Two-Station System with Different Distributions (Hypo-exponential: $c_{i}^{2}=0.64 \forall i$, Exponential: $c_{i}^{2}=1 \forall i$, and Cox-2: $c_{i}^{2}=4 \forall i$ ) with $\mu_{1}=0.7, \mu_{2}=0.5$

\begin{tabular}{rrrr|r|r|rr|rr}
\hline$K$ & $b_{i} \forall i$ & $M$ & $c_{i} \forall i$ & $|\mathcal{S}|$ & $E\left[T_{d}\right]$ & $\operatorname{Var}\left[T_{d}\right]$ & & $\operatorname{Var}\left[T_{s}\right]$ & \\
\hline 2 & 1 & 2 & 0.8 & 8 & 2.4483 & 3.4628 & 3.3553 & 3.4628 & 3.3553 \\
2 & 1 & 2 & 1 & 3 & 2.5952 & 5.3464 & 5.3464 & 5.3464 & 5.3464 \\
2 & 1 & 2 & 2 & 8 & 2.8699 & 22.892 & 22.9269 & 21.8136 & 21.8078 \\
2 & 2 & 3 & 0.8 & 12 & 2.2019 & 3.1198 & 2.9647 & 3.1198 & 2.9647 \\
2 & 2 & 3 & 1 & 4 & 2.3277 & 4.8288 & 4.8288 & 4.8288 & 4.8288 \\
2 & 2 & 3 & 2 & 12 & 2.655 & 21.832 & 21.8723 & 20.9544 & 21.0665 \\
2 & 3 & 4 & 0.8 & 16 & 2.1043 & 2.9558 & 2.779 & 2.9558 & 2.779 \\
2 & 3 & 4 & 1 & 5 & 2.2011 & 4.5341 & 4.5341 & 4.5341 & 4.5341 \\
2 & 3 & 4 & 2 & 16 & 2.5304 & 20.9857 & 21.0293 & 20.2158 & 20.3915 \\
2 & 4 & 5 & 0.8 & 20 & 2.0573 & 2.8706 & 2.6831 & 2.8706 & 2.6831 \\
2 & 4 & 5 & 1 & 6 & 2.1305 & 4.3559 & 4.3559 & 4.3559 & 4.3559 \\
2 & 4 & 5 & 2 & 20 & 2.4448 & 20.289 & 20.3445 & 19.5886 & 19.8161 \\
2 & 5 & 6 & 0.8 & 24 & 2.0325 & 2.8239 & 2.6307 & 2.8239 & 2.6307 \\
2 & 5 & 6 & 1 & 7 & 2.0875 & 4.2424 & 4.2424 & 4.2424 & 4.2424 \\
2 & 5 & 6 & 2 & 24 & 2.3805 & 19.7032 & 19.7807 & 19.0526 & 19.3315 \\
\hline \hline
\end{tabular}

Figure 12 depicts the computational time of the experiments reported in Tables 12,13 and 14. The computation time in these experiments ranges from milliseconds for a 2 -station system to 2 hours for a 4-station 5-buffer system on a personal computer with Intel Core CPU $3.4 \mathrm{GHz}$ with 8 GB RAM. Since the objective of this study is to introduce the methodology, only exploratory numerical results are obtained by using routines that are not optimized for speed. The computation time can be shortened by using sparse-matrix routines and by using a workstation with a higher working memory.

The system size this method can handle depends on the number of states (i.e. the size of the 
Table 13 Computational Results of a Three-Station System with an Exponential Distribution $\left(c_{i}^{2}=1 \forall i\right)$ and $\mu_{1}=0.7, \mu_{2}=0.5, \mu_{3}=0,9$ with the buffer levels $b_{i}=1,2, \ldots, 20 \forall i$

\begin{tabular}{rrr|r|r|rrr|rrr}
\hline$K$ & $b_{i} \forall i$ & $M$ & $|\mathcal{S}|$ & $E\left[T_{d}\right]$ & $\operatorname{Var}\left[T_{d}\right]$ & & & \\
\hline 3 & 1 & 3 & 10 & 2.5966 & 4.0873 & 5.0772 & 4.7236 & 5.5674 & 5.5674 & 5.5674 \\
3 & 2 & 5 & 18 & 2.2805 & 3.7279 & 4.5293 & 4.1966 & 4.4601 & 4.7100 & 4.4971 \\
3 & 3 & 6 & 25 & 2.1840 & 3.6016 & 4.3966 & 4.1575 & 4.2448 & 4.4848 & 4.3439 \\
3 & 4 & 8 & 36 & 2.1092 & 3.4893 & 4.2401 & 3.9848 & 4.1921 & 4.2922 & 4.1596 \\
3 & 5 & 9 & 46 & 2.0745 & 3.4365 & 4.1755 & 3.9956 & 4.1031 & 4.2029 & 4.1119 \\
3 & 6 & 11 & 60 & 2.0482 & 3.3958 & 4.1160 & 3.9261 & 4.0876 & 4.1323 & 4.0436 \\
3 & 7 & 12 & 73 & 2.0334 & 3.3722 & 4.0839 & 3.9505 & 4.0478 & 4.0927 & 4.0309 \\
3 & 8 & 14 & 90 & 2.0226 & 3.3555 & 4.0577 & 3.9193 & 4.0420 & 4.0629 & 4.0017 \\
3 & 9 & 15 & 106 & 2.0158 & 3.3445 & 4.0415 & 3.9442 & 4.0234 & 4.0443 & 4.0014 \\
3 & 10 & 17 & 126 & 2.0109 & 3.3371 & 4.0291 & 3.9292 & 4.0208 & 4.0307 & 3.9879 \\
3 & 11 & 18 & 145 & 2.0077 & 3.3318 & 4.0209 & 3.9505 & 4.0118 & 4.0218 & 3.9916 \\
3 & 12 & 20 & 168 & 2.0054 & 3.3283 & 4.0148 & 3.9432 & 4.0105 & 4.0153 & 3.9851 \\
3 & 13 & 21 & 190 & 2.0038 & 3.3257 & 4.0106 & 3.9600 & 4.0060 & 4.0109 & 3.9895 \\
3 & 14 & 23 & 216 & 2.0027 & 3.3240 & 4.0075 & 3.9563 & 4.0053 & 4.0077 & 3.9863 \\
3 & 15 & 24 & 241 & 2.0019 & 3.3227 & 4.0054 & 3.9692 & 4.0031 & 4.0055 & 3.9903 \\
3 & 16 & 26 & 270 & 2.0014 & 3.3219 & 4.0038 & 3.9673 & 4.0027 & 4.0039 & 3.9887 \\
3 & 17 & 27 & 298 & 2.0010 & 3.3212 & 4.0027 & 3.9769 & 4.0016 & 4.0028 & 3.9920 \\
3 & 18 & 29 & 330 & 2.0007 & 3.3208 & 4.0020 & 3.9759 & 4.0014 & 4.0020 & 3.9912 \\
3 & 19 & 30 & 361 & 2.0005 & 3.3205 & 4.0014 & 3.9829 & 4.0008 & 4.0014 & 3.9938 \\
3 & 20 & 32 & 396 & 2.0004 & 3.3203 & 4.0010 & 3.9825 & 4.0007 & 4.0010 & 3.9934 \\
\hline \hline
\end{tabular}

Table 14 Computational Results of a Four-Station System with a Cox-2 Distribution $\left(c_{i}^{2}=2 \forall i\right)$ and $\mu_{1}=0.7, \mu_{2}=0.5, \mu_{3}=0,9, \mu_{4}=0,7$ and $b_{i}=5$ buffers at each station $i=1,2,3,4$ with an increasing number of customers

\begin{tabular}{rrr|r|r|r|llll|llll}
\hline$K$ & $b_{i} \forall i$ & $M$ & $|\mathcal{S}|$ & $E\left[T_{d}\right]$ & $\operatorname{Var}\left[T_{d}\right]$ & & & & & \\
\hline 4 & 5 & 1 & 8 & 5.9683 & 37.2648 & 37.2648 & 37.2648 & 37.2648 & 37.2648 & 37.2648 & 37.2648 & 37.2648 \\
4 & 5 & 2 & 32 & 4.3803 & 34.3482 & 34.5416 & 34.4529 & 34.3982 & 32.2664 & 31.6890 & 32.8955 & 32.3160 \\
4 & 5 & 3 & 88 & 3.7776 & 31.3276 & 31.6701 & 31.5184 & 31.4193 & 29.7086 & 29.5865 & 30.2694 & 29.7999 \\
4 & 5 & 4 & 192 & 3.4361 & 28.9878 & 29.4527 & 29.2575 & 29.1176 & 27.6203 & 27.7891 & 28.1758 & 27.7498 \\
4 & 5 & 5 & 360 & 3.2070 & 27.1340 & 27.7063 & 27.4785 & 27.2987 & 25.9291 & 26.3163 & 26.5050 & 26.0939 \\
4 & 5 & 6 & 608 & 3.0389 & 25.6271 & 26.2968 & 26.0419 & 25.8230 & 24.5383 & 25.1036 & 25.1458 & 24.7349 \\
4 & 5 & 7 & 960 & 2.9085 & 22.3227 & 24.5705 & 23.8026 & 23.5455 & 23.3773 & 24.0941 & 24.0201 & 23.6016 \\
4 & 5 & 8 & 1,432 & 2.8179 & 21.9568 & 23.7758 & 23.0949 & 22.6959 & 21.4396 & 23.0637 & 22.5047 & 22.1247 \\
4 & 5 & 9 & 2,000 & 2.7516 & 21.5912 & 23.1401 & 22.5089 & 21.9523 & 21.2743 & 22.5346 & 22.0022 & 21.5877 \\
4 & 5 & 10 & 2,616 & 2.7023 & 21.2731 & 22.6320 & 22.0235 & 21.2993 & 21.0887 & 22.1055 & 21.5780 & 21.1241 \\
4 & 5 & 11 & 3,232 & 2.6655 & 21.0196 & 22.2288 & 21.6248 & 20.7200 & 20.9321 & 21.7650 & 21.2282 & 20.7323 \\
4 & 5 & 12 & 3,800 & 2.6387 & 20.8287 & 21.9123 & 21.2988 & 20.2585 & 20.8197 & 21.5026 & 20.9484 & 20.4050 \\
\hline \hline
\end{tabular}

transition rate matrix), the working memory of the computer used, and the efficiency of storing the transition rate matrix. In our case, with 8 GB RAM and an implementation in Visual Basic.NET, the limit is 11,000 states. 
Figure 12 Computation Time in Seconds with Logarithmic Scale Subject to the Number of States

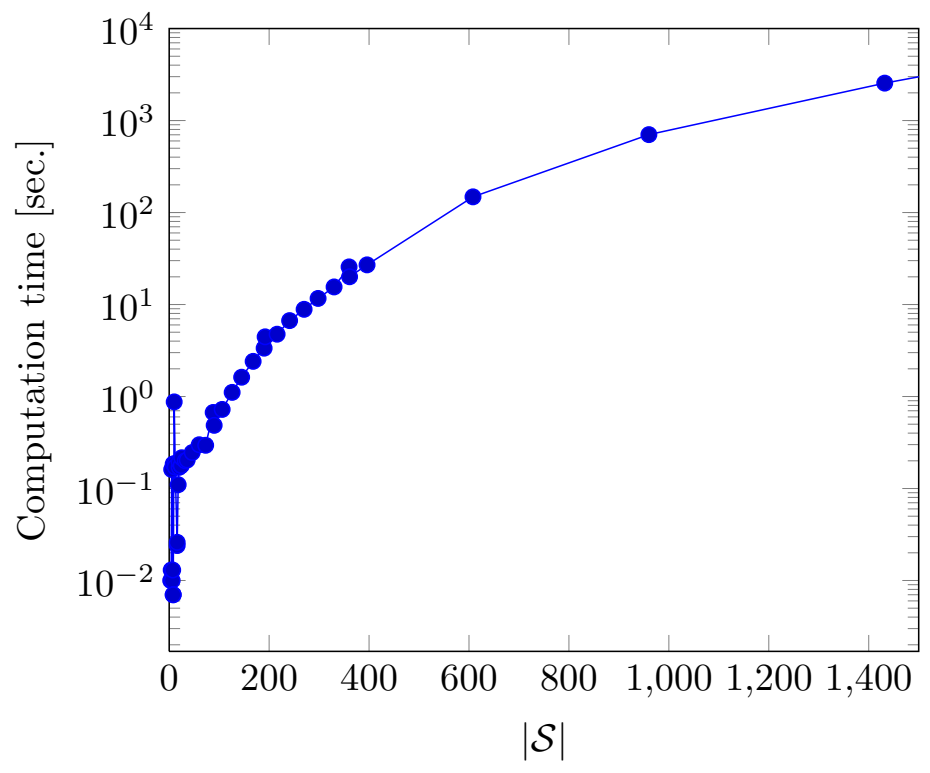

\section{Conclusion}

In this paper, we present a unified method to determine the inter-departure, inter-start, and cycle time distributions of closed queueing networks with phase-type servers subject to blocking. The two-stage method separates the model building and the analysis parts. Once the CTMC model of a production system is generated automatically in the first stage, the state space and the transition-rate matrix are used as the only inputs of the second stage.

The method extends the original state space and identifies the transitions that lead to a departure, to an arrival or to a completion of a workpiece. A first passage time analysis is used to determine the inter-event time distributions from the states where a part initiates an event until the next occurrence of the same event. For closed queueing networks with phase-type servers, the method yields the exact inter-departure, inter-start, and cycle time distributions and also their mean and variance by using only the transition-rate matrix and the indicator matrices that identify the transitions that lead to a departure, to an arrival, or to a completion of the process.

We use this methodology to model and analyze closed queueing networks subject to blocking that can be modeled as CTMC. We present an automated way to generate the state space. With this approach, we analyze queueing networks with servers that have exponential, Coxian-2, general Coxian, or other phase-type distributions. These distributions allow us to capture the first two moments of a given processing time distribution.

The same approach can also be used to analyze other queueing networks that can be modeled as CTMC. For example, the exact inter-departure and inter-start time distributions of reliable and unreliable production lines, production lines controlled with various control mechanisms, and 
production lines of other topologies such as open systems can be obtained using the same approach.

Since the method is based on analyzing the state space, the computational complexity increases rapidly with the number of stations and with the buffer capacities as all the state-space-based methods. Its ability to yield the exact inter-departure and inter-start time distributions for closed queueing networks that can be modeled as CTMCs makes it a viable method to analyze particular systems. Since the computational requirements increase rapidly for large systems, there is still a need to develop approximation methods. The proposed method allows evaluating the accuracy of these approximation methods by providing the exact distributions for different models. The exact results are more appropriate for evaluation of accuracy of approximation methods compared to results that can be obtained by using a simulation study.

This study can be extended in various ways. The proposed method yields the exact distribution for closed queueing networks with phase-type distributions. The method can be extended to tackle open queueing networks with phase-type servers. This approach can be used to analyze production lines. An approximation method can be developed to analyze larger systems by using the proposed methodology. The effectiveness of approximation methods that use two-station subsystems in decomposition approaches for production systems can be increased by investigating the best ways of representing the flow variability in a network by using the proposed method. Furthermore, the exact blocking and starvation time distributions can be determined by using the same method. Since delayed distributions with low variability model the service times well (Inman 1999), a method could be developed for systems where the processing times are delayed distributions.

We present this method as a general tool that yields the exact inter-departure, inter-start, and cycle time distributions for production systems that can be modeled as CTMCs.

\section{References}

Ayhan, H., Z. Palmoski, and S. Schlegel (2004). Cyclic queueing networks with subexponential service times. Journal of Applied Probability 41, 791-801.

Boxma, O. J. (1983). The cyclic queue with one general and one exponential server. Advances in Applied Probability 15(4), 857-873.

Boxma, O. J. and P. Donk (1982). On response time and cycle time distribution in a two-stage cyclic queue. Performance Evaluation 2(3), 181-194.

Boxma, O. J., F. P. Kelly, and A. G. Konheim (1984). The product form for sojourn time distributions in cyclic exponential queues. Journal of the Assiciation for Computing Machinery 31(1), 128-133. 
Buzacott, J. A., X. G. Liu, and J. G. Shanthikumar (1995). Multistage flow line analysis with the stopped arrival queue model. IIE Transactions $27(4), 444-455$.

Chow, W.-M. (1980). The cycle time distribution of exponential cyclic queues. Journal of the Association for Computing Machinery 27(2), 281-286.

Daduna, H. (1982). Passafe times for overtake-free paths in gordon-newell networks. Advances in Applied Probability $14(3), 672-686$.

Duenyas, I. and W. J. Hopp (1990). Estimating variance of output from cyclic exponential queueing systems. Queueing Systems: Theory and Application 7(3-4), 337-353.

Duenyas, I., W. J. Hopp, and M. L. Spearman (1993). Characterizing the output process of a CONWIP line with deterministic processing and random outages. Management Science 39(8), 975-988.

Gelenbe, E. (1975). On approximate computer system models. Journal of the Association for Computing Machinery 22(2), 261-269.

Gershwin, S. B. (1993). Variance of the output of a tandem production system. Queueing Networks with Finite Capacity, R. Onvural and I. Akyildiz (eds.), Proceedings of the Second International Conference on Queueing Networks with Finite Capacity.

Hendricks, K. B. (1992). The output processes of serial production lines of exponential machines with finite buffers. Operations Research 40(6), 1139-1147.

Hendricks, K. B. and J. O. McClain (1993). The output processes of serial production lines of general machines with finite buffers. Management Science 39(10), 1194-1201.

Inman, R. (1999). Empirical evaluation of exponential and independence assumptions in queueing models of manufacturing systems. Production and Operations Management 8, 409-432.

Kelly, F. and P. K. Pollett (1983). Sojourn times in closed queuing networks. Advances in Applied Probability $15(3), 638-656$.

Kim, D. S. and J. M. Alden (1997). Estimating the distribution and variance of time to produce a fixed lot size given deterministic processing times and random down times. International Journal of Production Research 35(12), 3405-3414.

Lagershausen, S. (2012). Performance Analysis of Closed Queueing Networks. Lecture Notes in Economics and Mathematical Systems (663). Springer.

Li, J. and S. M. Meerkov (2000). Production variability in manufacturing systems: Bernoulli reliability case. Annals of Operations Research 93(1-4), 299-324. 
Manitz, M. (2005). Leistungsanalyse von Montagesystemen mit stochastischen Bearbeitungszeiten. Ph. D. thesis, Universität zu Köln.

Manitz, M. and H. Tempelmeier (2012). The variance of inter-departure times of the output of an assembly line with finite buffers, converging flow of material, and general service times. OR Spectrum 34(1), 273-291.

Miltenburg, G. J. (1987). Variance of the number of units produced on a transfer line with buffer inventories during a period of length t. Naval Research Logistics 34(6), 811-822.

Neuts, M. F. (1995). Matrix-Geometric Solutions in Stochastic Models: An Algorithmic Approach (2 ed.). Mineola, N. Y.: Dover Publications Inc.

Schassberger, R. and H. Daduna (1983). The time for a round trip in a cycle of exponential queues. Journal of the Association for Computing Machinery 30(1), 146-150.

Shi, C. and S. B. Gershwin (2011). Part waiting time distribution in a two-machine line. Proceedings of the IXth International Conference on Stochastic Models of Manufacturing and Service Operations, $261-268$

Tan, B. (1998). An analytical formula for variance of output from a series-parallel production system with no interstage buffers and time-dependent failures. Mathematical and Computer Modelling 27(6), 95-112.

Tan, B. (1999a). Asymptotic variance rate of the output of a transfer line with no buffer storage and cycle-dependent failures. Mathematical and Computer Modelling 29(7), 97-112.

Tan, B. (1999b). Variance of the output as a function of time: Production line dynamics. European Journal of Operational Research 117(3), 470-484.

Tan, B. (2000). Asymptotic variance rate of the output in production lines with finite buffers. Annals of Operations Research 93(41), 385-403.

Tan, B. (2013). Modeling and analysis of output variability in discrete material flow production systems. In B. Tan and J. M. Smith (Eds.), Handbook of Stochastic Models and Analysis of Manufacturing System Operations, Volume 192 of International Series in Operations Research 83 Management Science, pp. 287-311. New York: Springer.

Wu, K. and L. McGinnis (2013). Interpolation approximations for queues in series. IIE Transactions 45(3), 273-290. 